\title{
Meta-analysis of long-term joint structural deterioration in minimally treated patients with rheumatoid arthritis
}

Jeroen P. Jansen ${ }^{1,4^{*}}$, Maria-Cecilia Vieira ${ }^{2}$, John D. Bradley ${ }^{3}$, Joseph C. Cappelleri ${ }^{3}$, Samuel H. Zwillich ${ }^{3}$ and Gene V. Wallenstein ${ }^{3}$

\begin{abstract}
Background: Rheumatoid arthritis (RA) is a chronic autoimmune disease characterized by inflammation and joint structural deterioration. Driven by recent expectations that patients in clinical trials randomized to placebo should be 'rescued' with active therapy within 6 months of starting treatment, the relative benefit of arresting joint damage with biologic agents beyond this period is unclear. With longer-term evidence of the rate of joint deterioration with minimal treatment, the efficacy of biologic agents and novel treatments might be projected beyond the placebo-controlled phase observed in clinical trials. The aim of this study was to estimate radiographic structural deterioration over time in patients with moderate-to-severe RA minimally treated with DMARDs.

Methods: A literature review identified evidence of joint structural deterioration in patients with (DMARD-IR population) and without (non-DMARD-IR population) a history of inadequate response to DMARDs. Patients were minimally treated with one non-biologic DMARD or palliative care (non-DMARD-IR population only). Outcomes of interest were the (modified) Total Sharp Score (TSS) and subscales (Erosion Subscore [ES] and Joint Space Narrowing [JSN] Subscore), and Larsen score. Pooled joint-deterioration curves over time were obtained with meta-analysis models.

Results: Mean change from baseline in TSS increased in the DMARD-IR population from 1.14 (95\% credible interval $[\mathrm{Crl}] \mathrm{0.66}, 1.67)$ to $9.84(5.68,14.46)$ at Weeks 12 and 104, respectively, and a non-linear increase of $1.56(0.79,2.34)$ and $5.13(-1.35,11.67)$ in the non-DMARD-IR population. At the same time points, mean changes $(95 \% \mathrm{Crl})$ were $0.51(0.27,0.83)$ and $4.43(2.38,7.21)$ for ES and $0.36(0.09,0.67)$ and $3.14(0.80,5.78)$ for JSN in the DMARD-IR population, whereas corresponding changes in the non-DMARD-IR population were $0.69(0.31,1.12)$ and $2.93(0.92,5.02)$, and $0.29(0.17,0.44)$ and $2.55(1.45,3.80)$, respectively. Larsen scores were only available for the non-DMARD-IR population, with mean changes $(95 \% \mathrm{Crl})$ of $0.08(0.04,0.11)$ and $0.65(0.36,0.96)$ at Weeks 12 and 104, respectively.
\end{abstract}

Conclusion: Minimal treatment of RA with one non-biologic DMARD results in deterioration of joint structure in patients with or without a history of inadequate response to non-biologic DMARDs.

Keywords: Joint erosions, Longitudinal studies, Meta-analysis, Radiography, Rheumatoid arthritis

\footnotetext{
* Correspondence: jeroen.jansen@tufts.edu

${ }^{1}$ Tufts University School of Medicine, Boston, MA, USA

${ }^{4} 1714$ Stockton Street, 3rd floor, San Francisco, CA 94133, USA

Full list of author information is available at the end of the article
} 


\section{Background}

Rheumatoid arthritis (RA) is a common chronic inflammatory joint disorder. Without treatment most patients with RA become severely disabled. The goals of RA treatment are to reduce disease activity, reduce or inhibit the rate of joint damage and, if possible, achieve remission. Current pharmacologic therapies include traditional disease-modifying anti-rheumatic drugs (DMARDs) and biologic agents [1-3].

Biologic agents have been shown to inhibit radiographic joint destruction in patients with an inadequate response to non-biologic DMARDs. Driven by recent expectations that patients in clinical trials randomized to placebo should be 'rescued' with active therapy within 6 months of starting treatment, the relative benefit of arresting joint damage with biologic agents beyond this period is unclear. With longer-term evidence of the rate of joint deterioration with placebo or minimal treatment, the efficacy of biologic agents and novel treatments might be projected beyond the placebo-controlled phase observed in clinical trials.

The objective of the current study was to estimate radiographic joint destruction over time with minimal treatment among the following populations of biologic DMARD-naïve RA patients: (1) moderate-to-severe RA patients with a history of inadequate response to nonbiologic DMARDs who were treated with one (other) non-biologic DMARD; and (2) moderate-to-severe RA patients without a history of inadequate response to a DMARD, who received palliative care (non-steroidal anti-inflammatory drugs [NSAIDs], analgesics, low-dose glucocorticoids) or were being minimally treated with one non-biologic DMARD. The first population was termed the "DMARD-IR population" and the second population the "non-DMARD-IR population". The evidence for this analysis was obtained by means of a systematic literature review.

\section{Methods}

\section{Study identification and selection}

A systematic literature search was performed to identify studies that provided information concerning joint structural deterioration among minimally treated RA patients. MEDLINE ${ }^{\oplus}$ and EMBASE $^{\oplus}$ databases were searched simultaneously for articles published in English, French, or German, from 1970 to October 2009, with a predefined search strategy. Search terms included a combination of free text and thesaurus terms related to RA, NSAIDs, glucocorticoids, non-biologic DMARDs, clinical trials, and observational studies. (See Additional file 1 for details of the search strategy.) The relevance of each citation identified from the databases was based on the title and abstract according to the predefined selection criteria outlined below:

\section{Populations of interest}

DMARD-IR, i.e., adult RA patients naïve to biologic DMARDs with a history of inadequate response to one or more non-biologic DMARDs; and non-DMARD-IR, i.e., adult RA patients naïve to biologic DMARDs without a history of inadequate response to a non-biologic DMARD. The non-DMARD-IR population could include both non-biologic DMARD-naïve (completely DMARD-naïve) and non-naïve (non-biologic DMARDexperienced) patients.

\section{Interventions}

NSAIDs, glucocorticoids, and single non-biologic DMARDs, including methotrexate (MTX), azathioprine (AZA), sulfasalazine (SSZ), leflunomide (LEF), ciclosporin A (CSA), hydroxychloroquine (HCQ), minocycline, D-penicillamine, and gold salts.

\section{Outcomes}

Radiographic measures of joint deterioration: Larsen score (0-200 points range) [4, 5] and Total Sharp Score (TSS) (0-448), plus two TSS subscores (Erosion Subscore [ES] (0-280) and Joint Space Narrowing [JSN] (0-168) Subscore) [6-10].

\section{Study design}

Randomized controlled trials (RCTs), and prospective and retrospective observational cohort studies. Only study arms concerning the interventions of interest were included.

Publications were obtained, if available, for any abstracts that potentially met the selection criteria. Based on these full-text reports, two reviewers evaluated whether each study met the selection criteria and any disagreements were resolved in a consensus meeting.

\section{Data extraction}

For each of the selected studies that reported sufficient follow-up data, details were extracted from the relevant study arms on study design, population characteristics, interventions, and the outcomes of interest, i.e., the (modified) TSS and its two subscores (ES and JSN). Data were extracted into a study database according to the Preferred Reporting Items for Systematic Reviews and Meta-analyses (PRISMA) 2009 [11]. Mean change from baseline (CFB) in the outcomes of interest was extracted from tables, text, or graphs. If not reported, CFBs were calculated as the difference between reported follow-up and baseline values. Corresponding standard errors were extracted directly or calculated indirectly based on the following data (if available): reported standard deviation (SD) with sample size, $95 \%$ confidence interval, or $p$-values (in this order of preference). 
Larsen scores were not consistently evaluated or reported. Different numbers and sets of joints were evaluated in the various studies, including hands and feet, or hands, feet, and wrists, and many studies did not report which or how many joints were evaluated. Moreover, some studies reported the total scores and some reported an average of scores per joint. Consequently, the analyses were based on standardized mean CFB in Larsen score, calculated as the reported CFB divided by the corresponding SD of this change.

\section{Meta-analysis of joint structural deterioration over time} Mean CFB in TSS, ES, JSN, and the standardized Larsen scores obtained from the selected studies were combined with Bayesian random-effects meta-analysis models to estimate joint deterioration over time for the DMARDIR and non-DMARD-IR populations [12]. Any study that did not explicitly state whether or not patients had previously shown an inadequate response to DMARDs was assigned to the non-DMARD-IR population. Depending on the availability of data by endpoint and population, two sets of analyses were performed. In the first series of analyses, all non-biologic-DMARDs were considered as one group and the development of the outcomes of interest was estimated. Studies evaluating only NSAIDs were not combined with studies evaluating DMARDs. In the second series of analyses, the development of the outcome over time was compared among individual DMARD (e.g., MTX, LEF, and AZA) using only data from comparative studies. All analyses provided curves reflecting the pooled mean CFB in TSS, ES, JSN, and the standardized Larsen score over time, along with their respective $95 \%$ credible intervals (95\% CrIs).

Within the Bayesian framework, analyses consisted of data, likelihood, parameters, and a model. Bayesian methods involve a formal combination of a prior probability distribution (that reflects a prior belief of the possible values of the parameters of interest) with a likelihood distribution based on the observed data, to obtain a posterior probability distribution of the parameters of interest [13]. A normal likelihood distribution was assumed.

We opted for statistical models that assume that outcomes develop over time in a linear fashion, as well as models that anticipate that outcomes can develop in a non-linear fashion over time [14-16]. The advantage of the used meta-analysis models is that all available data points of each study included in the analysis are captured, even if time points are not the same across studies, and (non-) linear trends of the development of outcomes over time are estimated [12]. Details of the meta-analysis models are provided in Additional file 2. Model 1 and 2 were used to estimate the development over time where all non-biologic DMARDs were grouped. Model 3 and 4 were used for the comparative analysis of different DMARDS. The deviance information criterion (DIC) provides a measure of model fit that penalizes model complexity and was used to compare the different models $[17,18]$. The model with the lowest DIC and, therefore the model with the "best fit", was considered the most appropriate.

To avoid prior beliefs influencing the results of the model, non-informative prior distributions were used. Prior distributions of all model parameters were normal distributions with a mean of 0 and a variance of $10^{4}$, except for heterogeneity, which was a uniform distribution with a range of $0-10$. With such a "flat" prior, it is assumed that, in advance of the actual data, any parameter value is "equally" likely. As a consequence, posterior results are not influenced by the prior distribution but are driven by the data. The result of the Bayesian analysis is a (joint) posterior distribution for the model parameters of interest. The model parameters were estimated using a Markov chain Monte Carlo method as implemented in the WinBUGS software package [19].

\section{Results \\ Study selection}

The study selection process, including the reasons for exclusion, is summarized in Fig. 1. The literature search identified 2076 potentially relevant studies, although the first review excluded 1892 (91 \%) of these. The full-text review of the 184 remaining studies excluded another 111 studies. Of the 73 articles meeting the selection criteria, another 29 studies were excluded because of insufficient data on the outcomes of interest during follow-up. Overall, 44 studies were included [20-63].

\section{Study characteristics}

Information on key study and patient characteristics are presented in Tables 1 and 2. All 44 studies were RCTs, except for one retrospective [31] and two prospective cohort studies [33, 52], and were published between 1982 and 2009, with follow-up periods ranging from 24 weeks to 2 years. Twelve studies concerned the DMARD-IR population [20-31] and the remaining 32 studies concerned the non-DMARD-IR population [32-63]. Only two studies provided data on the Larsen score for the DMARD-IR population, while 10 provided data on the (modified) TSS in this population. For the non-DMARD-IR population, 17 studies provided data on Larsen score only, 14 studies provided data on the (modified) TSS, and one study provided data on both. As some of the studies contributed data from more than one arm (three studies in the DMARD-IR population $[21,22,31]$ and 13 


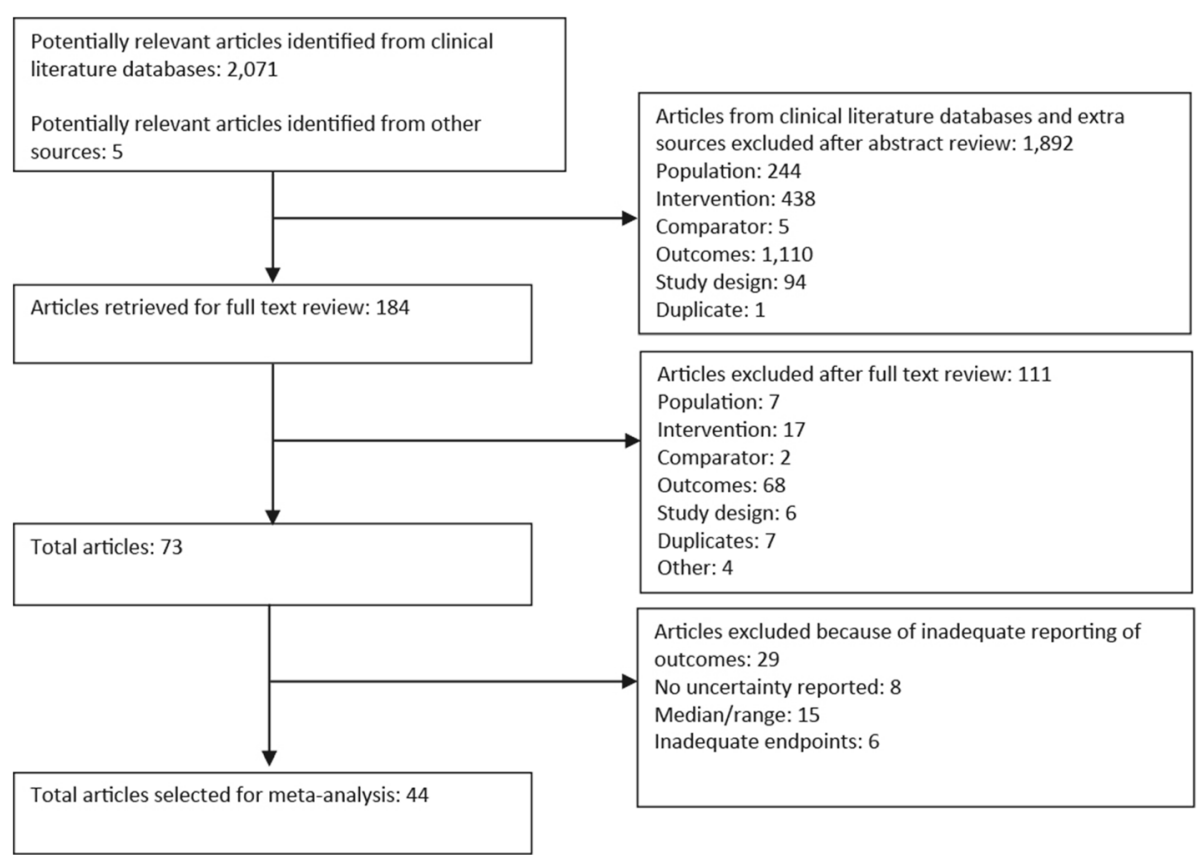

Fig. 1 Flow diagram of study selection

studies in the non-DMARD-IR population [35, 36, 38, $41,48,50,51,54,57,59,61-63])$, the total number of treatment arms included in the analyses was 63: 16 for DMARD-IR and 47 for non-DMARD-IR. Among the 47 arms that formed the non-DMARD-IR population, only 12 included patients who had been previously exposed to DMARDs. Hence, the majority of patients in this population could be considered DMARD-naïve.

The patients received the following treatments $g$ : MTX (in 12 treatment arms across the studies); AZA (2); SSZ (1); and gold salts (1) for the DMARD-IR population; and MTX (16); gold salts (9); SSZ (5); LEF (four 4); CSA (4); HCQ (1); D-penicillamine (1); antimalarials, D-penicillamine, SSZ, or gold salts (2); any one of a list of non-biologic DMARDs (4); and NSAIDs (4) for the non-DMARD-IR population. The number of patients included ranged from 29 to 228 for DMARD-IR arms and from 20 to 501 for nonDMARD-IR arms.

\section{Patient characteristics}

The DMARD-IR and the non-DMARD-IR populations showed comparable distributions for gender, age, baseline C-reactive protein (CRP) level, and erythrocyte sedimentation rate (ESR). On average, $73 \%$ of the patients were women in the DMARD-IR studies versus $70 \%$ in the non-DMARD-IR studies; the average ages were 54 and 52 years, respectively. The median of the reported baseline CRP level was $2.2 \mathrm{mg} / \mathrm{dL}$ across the DMARD-
IR studies and $2.6 \mathrm{mg} / \mathrm{dL}$ across the non-DMARD-IR studies. The median of the reported baseline ESR was $43 \mathrm{~mm} / \mathrm{h}$ across DMARD-IR studies and $44 \mathrm{~mm} / \mathrm{h}$ across non-DMARD-IR studies. There was a large variation in baseline CRP level and ESR across the nonDMARD-IR studies. As expected, the disease duration was skewed and longer among the DMARD-IR patients (median of 88 months) versus the non-DMARD-IR patients (median of 15 months). For the DMARD-IR population, the median of the reported baseline Health Assessment Questionnaire (HAQ) score across the studies was 1.7; the HAQ scale ranges from zero (no disability) to three (completely disabled). The median baseline TSS for the DMARD-IR population was 53. For the non-DMARD-IR population the median of the reported HAQ scores across studies was 1.0 and the median TSS was 11.9.

\section{Joint structural deterioration over time in the DMARD-IR population}

The mean CFB in TSS within the DMARD-IR population, as obtained from the individual studies, is presented in Fig. 2. These results were combined with a random-effects meta-analysis (Additional file 2, Model 1), where the change in TSS over time developed in a linear fashion from 1.14 at Week 12 to 9.84 at Week 104 (Table 3). There was a high probability that continuation of treatment with any one non-biologic DMARD in the setting of inadequate 
Table 1 Key study characteristics

\begin{tabular}{|c|c|c|c|c|c|c|c|c|}
\hline Study [Ref] & Study design & Follow-up period & $\begin{array}{l}\text { Reassignment in } \\
\text { studies of biologics }\end{array}$ & Larsen & JSN & ES & TSS & $\begin{array}{l}\text { Outcomes of } \\
\text { interest }\end{array}$ \\
\hline \multicolumn{9}{|l|}{ DMARD-IR population } \\
\hline Breedveld et al. 2004 [20] & $\mathrm{RCT}$ & NA & $\begin{array}{l}\text { No reassignment; MTX dose } \\
\text { increase for non-responders }\end{array}$ & No & No & No & Yes & Modified TSS \\
\hline Hamdy et al. 1987 [21] & Double-blind RCT & 1.5 years & NA & No & No & No & Yes & TSS \\
\hline Jeurissen et al. 1991 [22] & Double-blind RCT & 48 weeks & NA & No & No & No & Yes & Modified TSS \\
\hline Keystone et al. 2004 [23] & Double-blind RCT & 1 year & $\begin{array}{l}\text { No reassignment; MTX dose } \\
\text { increase for non-responders }\end{array}$ & No & Yes & Yes & Yes & Modified TSS \\
\hline Keystone et al. 2008 [24] & Double-blind RCT & 1 year & No reassignment & No & Yes & Yes & Yes & Modified TSS \\
\hline Lipsky et al. 2000 [25] & $\mathrm{RCT}$ & 1 year & No reassignment & No & Yes & Yes & Yes & Modified TSS \\
\hline Maini et al. 2004 [26] & Double-blind RCT & 2 years & No reassignment & No & Yes & Yes & Yes & Modified TSS \\
\hline Sany et al. 1990 [27] & $\mathrm{RCT}$ & 2 years & NA & Yes & No & No & No & Larsen scores \\
\hline Smolen et al. 2009 [28] & $\mathrm{RCT}$ & 2 years & No reassignment & No & Yes & Yes & Yes & Modified TSS \\
\hline van der Heijde et al. 2006 [29] & Double-blind RCT & 2 years & No reassignment & No & Yes & Yes & Yes & Modified TSS \\
\hline van Holten et al. 2005 [30] & Double-blind RCT & 24 weeks & NA & No & No & No & Yes & Modified TSS \\
\hline Wick et al. 2005 [31] & Retrospective cohort & NA & NA & Yes & No & No & No & Larsen scores \\
\hline \multicolumn{9}{|l|}{ Non-DMARD-IR population } \\
\hline Bathon et al. 2000 [32] & $\mathrm{RCT}$ & 1 year & No reassignment & No & Yes & Yes & Yes & Modified TSS \\
\hline Boiardi et al. 1999 [33] & Prospective cohort & 1 year & NA & Yes & No & No & No & Larsen scores \\
\hline Breedveld et al. 2006 [34] & Double-blind RCT & 2 years & $\begin{array}{l}\text { No reassignment; } \\
\text { MTX dose increase } \\
\text { for non-responders }\end{array}$ & No & Yes & Yes & Yes & Modified TSS \\
\hline Choy et al. 2008 [35] & Double-blind RCT & 2 years & NA & Yes & No & No & No & Larsen scores \\
\hline Cohen et al. 2001 [36] & Double-blind RCT & 2 years & NA & No & Yes & Yes & Yes & Modified TSS \\
\hline Cohen et al. 2008 [37] & Double-blind RCT & 1 year & NA & No & Yes & Yes & No & Modified TSS \\
\hline Emery et al. 2000 [38] & Double-blind RCT & 2 years & NA & Yes & No & No & No & Larsen scores \\
\hline Emery et al. 2008 [39] & Double-blind RCT & 1 year & No reassignment & No & No & No & Yes & Modified TSS \\
\hline Emery et al. 2009 [40] & Double-blind RCT & 2 years & No reassignment & No & Yes & Yes & Yes & Modified TSS \\
\hline Ferraccioli et al. 1996 [41] & Open-label RCT & 1 year & NA & Yes & No & No & No & Larsen scores \\
\hline Ferraccioli et al. 1997 [42] & Open-label RCT & 1 year & NA & Yes & No & No & No & Larsen scores \\
\hline Goldbach-Mansky et al. 2009 [43] & $\mathrm{RCT}$ & 24 weeks & NA & No & Yes & Yes & Yes & Modified TSS \\
\hline Hannonen et al. 1993 [44] & Double-blind RCT & 48 weeks & NA & No & Yes & Yes & Yes & Modified TSS \\
\hline Hetland et al. 2006 [45] & Double-blind RCT & 1 year & NA & Yes & No & No & No & Larsen scores \\
\hline Hetland et al. 2008 [46] & Double-blind RCT & 2 years & NA & No & Yes & Yes & Yes & Modified TSS \\
\hline Landewé et al. 2002 [47] & Double-blind RCT & 1 year & NA & No & No & No & Yes & Modified TSS \\
\hline Larsen et al. 2001 [48] & Double-blind RCT & 24 weeks & NA & Yes & No & No & No & Larsen scores \\
\hline Maravic et al. 1999 [49] & Open-label RCT & 1 year & NA & Yes & No & No & Yes & $\begin{array}{l}\text { Larsen scores } \\
\text { and TSS }\end{array}$ \\
\hline Marchesoni et al. 2002 [50] & $\begin{array}{l}\text { First phase was open } \\
\text { and uncontrolled; } \\
\text { second phase was } \\
\text { single-blind RCT }\end{array}$ & 2 years & NA & Yes & Yes & Yes & Yes & $\begin{array}{l}\text { Larsen scores } \\
\text { and TSS }\end{array}$ \\
\hline Peltomaa et al. 1995 [51] & $\mathrm{RCT}$ & 1 year & NA & Yes & No & No & No & Larsen scores \\
\hline Sanmarti et al. 2003 [52] & Prospective cohort & 1 year & NA & Yes & No & No & No & Larsen scores \\
\hline Sarzi-Puttini et al. 2005 [53] & $\mathrm{RCT}$ & 1 year & NA & Yes & No & No & No & Larsen scores \\
\hline Scott et al. 1985 [54] & $\mathrm{RCT}$ & 1 year & NA & Yes & No & No & No & Larsen scores \\
\hline Scott et al. 1989 [55] & $\mathrm{RCT}$ & 1 year & NA & Yes & No & No & No & Larsen scores \\
\hline
\end{tabular}


Table 1 Key study characteristics (Continued)

\begin{tabular}{|c|c|c|c|c|c|c|c|c|}
\hline Scott et al. 1990 [56] & RCT & 2 years & NA & Yes & No & No & No & Larsen scores \\
\hline Smith et al. 1982 [57] & Open-label RCT & 2 years & NA & Yes & No & No & No & Larsen scores \\
\hline St Clair et al. 2004 [58] & $\mathrm{RCT}$ & 2 years & No reassignment & No & Yes & Yes & Yes & Modified TSS, \\
\hline Strand et al. 1999 [59] & Double-blind RCT & 1 year & NA & No & Yes & Yes & Yes & Modified TSS \\
\hline Svensson et al. 2003 [60] & Double-blind RCT & 1.5 years & NA & Yes & No & No & No & Larsen scores \\
\hline Svensson et al. 2005 [61] & Open-label RCT & 2 years & NA & No & Yes & Yes & Yes & Modified TSS \\
\hline van Riel et al. 1986 [62] & Single-blind RCT & 1 year & NA & Yes & No & No & No & $\begin{array}{l}\text { Modified Larser } \\
\text { scores }\end{array}$ \\
\hline Wassenberg et al. 2005 [63] & Double-blind RCT & 2 years & NA & No & Yes & Yes & Yes & Modified TSS \\
\hline
\end{tabular}

DMARD disease-modifying anti-rheumatic drug, DMARD-IR patient population with moderate-to-severe RA with a history of inadequate response to non-biologic DMARDs who are currently treated with one (other) non-biologic DMARD, ES Erosion Subscore of TSS, JSN Joint Space Narrowing Subscore of TSS, MTX methotrexate, NA not available, non-DMARD-IR patient population with moderate to severe RA without a history of inadequate response to a DMARD who are currently receiving palliative care (non-steroidal anti-inflammatory drugs, analgesics, low-dose glucocorticoids) or are being minimally treated with one non-biologic DMARD, $R A$ rheumatoid arthritis, Ref reference, $R C T$ randomized controlled trial, TSS modified Total Sharp Score

response would result in deterioration of the joint structure over time.

Table 3 also presents the results of the analysis (Additional file 2, Model 3) that compared joint deterioration as observed with MTX and AZA. Continuation of treatment with AZA was associated with greater joint deterioration than continuation of treatment with MTX in this DMARD-IR population.

The progression in ES extracted from the individual studies and the pooled results $(0.51$ at Week 12 and 4.43 at Week 104) obtained with the meta-analysis (Additional file 2, Model 1) are presented in Fig. 3. There was a $98 \%$ to $100 \%$ chance that ES would deteriorate over time when DMARD-IR patients received minimal treatment with a non-biologic DMARD (Table 3). As inferred from the comparative analysis, a greater rate of deterioration was expected with AZA than with MTX.

When DMARD-IR patients were treated with MTX alone mean changes in JSN were 0.36 at Week 12 and 3.14 at Week 104 (Fig. 4; Table 3).

For joint deterioration as measured with the Larsen score, only two studies with sufficient data were available for the DMARD-IR population [27, 31]. As neither study reported repeated intermediate observations, no metaanalysis model for change over time was estimated. At 24 weeks, a deterioration of 0.52 was observed with MTX [27]. In the other study the deterioration varied from 0.53 points with SSZ to 1.06 with gold salts at 52 weeks [31].

\section{Joint structural deterioration over time in the non-DMARD-IR population}

The rate of deterioration in the non-DMARD-IR population was not as great as for the DMARD-IR patients. The progression of the TSS for the non-DMARD-IR population is presented in Fig. 5 and Table 4. Individual study results were combined with a random-effects meta-analysis model, where the change in TSS from baseline developed in a non-linear fashion (fractional polynomial with $\mathrm{p} 1=\mathrm{p} 2=1$. Additional file 2 , Model 2) and shows an increase in TSS from 1.56 at 12 weeks to 5.13 at 104 weeks. Up to at least 104 weeks, there was at least a $94 \%$ chance that continuing treatment with one DMARD would result in deterioration of the joint structure in the non-DMARD-IR population (Table 4). An analysis (Additional file 2, Model 4) comparing LEF and MTX based on two head-to-head RCTs indicated a similar rate of deterioration with LEF and MTX $[37,59]$.

For ES, the individual study results were also combined with a random-effects non-linear meta-analysis model (fractional polynomial with $\mathrm{p} 1=1$ and $\mathrm{p} 2=0.5$; Additional file 2, Model 2) and showed that ES worsened over time from 0.69 at Week 12 to 2.93 at Week 104 when non-DMARD-IR patients continued to receive one traditional DMARD (Fig. 6; Table 4). Comparative analysis showed no difference in rate of deterioration was expected between LEF and MTX (Additional file 2, Model 4).

For the JSN and the Larsen scores, linear metaanalysis models were appropriate to reflect the deterioration up to 104 weeks (Fig. 7 and Table 4; Additional file 2, Model 1). One study evaluated treatment with NSAIDs only and reported a mean standardized change from baseline in the Larsen score of 0.01 up to 52 weeks [54].

\section{Discussion}

In this study, the development of joint structural deterioration among minimally treated patients with moderate-to-severe RA was estimated based on currently available published data. Estimates were obtained for two populations: a DMARD-IR population that consisted of patients who showed previous inadequate response with non-biologic DMARDs, and a nonDMARD-IR population that consisted of both non- 
Table 2 Patient characteristics at baseline

\begin{tabular}{|c|c|c|c|c|c|c|c|c|c|c|c|c|c|c|c|}
\hline \multirow{2}{*}{$\begin{array}{l}\text { Study [Ref] } \\
\text { Patient type }\end{array}$} & \multirow{2}{*}{$\begin{array}{l}\text { Treatment } \\
\text { (No. patients, } \\
\text { ITT) }\end{array}$} & \multirow[t]{2}{*}{ Female, \% } & \multirow{2}{*}{$\begin{array}{l}\text { Mean age } \\
(\mathrm{SD}) \text {, years }\end{array}$} & \multirow{2}{*}{$\begin{array}{l}\mathrm{RF}+, \\
\%\end{array}$} & \multirow{2}{*}{$\begin{array}{l}\text { Mean disease } \\
\text { duration (SD), } \\
\text { months }\end{array}$} & \multirow{2}{*}{$\begin{array}{l}\text { Mean CRP } \\
\text { (SD), } \mathrm{mg} / \mathrm{dL}\end{array}$} & \multirow{2}{*}{$\begin{array}{l}\text { Mean ESR (SD), } \\
\mathrm{mm} / \mathrm{h}\end{array}$} & \multicolumn{8}{|c|}{ Mean score (SD) } \\
\hline & & & & & & & & $\mathrm{HAQ}(0-3)$ & DAS-28 & SJC & TJC & TSS & JSN & ES & Larsen \\
\hline \multicolumn{16}{|l|}{ DMARD-IR population } \\
\hline $\begin{array}{l}\text { Breedveld et al. } 2004 \text { [20] } \\
\text { DMARD-experienced }\end{array}$ & $\operatorname{MTX}(n=88)$ & 78 & $54^{a}$ & NA & 100.8 & $2.6^{\mathrm{a}}$ & $42^{\mathrm{a}}$ & $1.8^{\mathrm{a}}$ & NA & $20^{\mathrm{a}}$ & $31^{\mathrm{a}}$ & $52^{\mathrm{a}}$ & $28^{a}$ & $24^{a}$ & NA \\
\hline \multirow{2}{*}{$\begin{array}{l}\text { Hamdy et al. } 1987 \text { [21] } \\
\text { DMARD-experienced }\end{array}$} & AZA $(n=21)$ & NA & NA & NA & NA & NA & 44.8 & NA & NA & 21.6 & NA & 53.1 & NA & NA & NA \\
\hline & $\operatorname{MTX}(n=21)$ & NA & NA & NA & NA & NA & 41.5 & NA & NA & 22.2 & NA & 44.5 & NA & NA & NA \\
\hline \multirow{2}{*}{$\begin{array}{l}\text { Jeurissen et al. } 1991 \text { [22] } \\
\text { DMARD-experienced }\end{array}$} & AZA $(n=33)$ & 52 & $56(9)$ & NA & $112.8(67.2)$ & NA & $60.2(27.9)$ & NA & NA & $18.7(7.0)$ & $23.0(10.7)$ & 60.5 & NA & NA & NA \\
\hline & $\operatorname{MTX}(n=31)$ & 84 & $57(10)$ & NA & $153.6(120.0)$ & NA & 57.5 (27.9) & NA & NA & $18.8(7.2)$ & $22.5(10.1)$ & 62.7 & NA & NA & NA \\
\hline $\begin{array}{l}\text { Keystone et al. } 2004 \text { [23] } \\
\text { DMARD-experienced }\end{array}$ & MTX $(n=200)$ & 73 & $56(12)$ & 89.5 & 130.8 (105.6) & $1.8(2.1)$ & NA & $1.5(0.6)$ & NA & $19(9.5)$ & $28.1(13.8)$ & $66.4(47.4)$ & $29.2(24.5)$ & $37.2(25.8)$ & NA \\
\hline $\begin{array}{l}\text { Keystone et al. } 2008 \text { [24] } \\
\text { MTX-experienced }\end{array}$ & $\operatorname{MTX}(n=199)$ & 84 & $52(11)$ & 82.8 & $74.4(52.8)$ & $1.6^{\mathrm{a}}$ & NA & $1.7(0.6)$ & $7.0^{\mathrm{a}}$ & $21.2(9.7)$ & $29.8(13.0)$ & NA & NA & NA & NA \\
\hline $\begin{array}{l}\text { Lipsky et al. } 2000 \text { [25] } \\
\text { DMARD-IR }\end{array}$ & $\operatorname{MTX}(n=88)$ & 80 & $51(12)$ & 77.0 & $132.0(96.0)$ & $4.0(4.2)$ & $49.0(25.0)$ & $1.7(0.6)$ & NA & $21(12.0)$ & $31.0(18.0)$ & $82.0(77.0)$ & NA & NA & NA \\
\hline $\begin{array}{l}\text { Maini et al. } 2004 \text { [26] } \\
\text { DMARD-experienced }\end{array}$ & $\operatorname{MTX}(n=88)$ & 78 & 54 & NA & NA & 2.6 & NA & 1.8 & NA & 20.0 & 31.0 & NA & 28.3 & 23.5 & NA \\
\hline $\begin{array}{l}\text { Sany et al. } 1990 \text { [27] } \\
\text { DMARD-experienced }\end{array}$ & $\operatorname{MTX}(n=41)$ & 88 & $54(27-78)^{b}$ & 82.9 & 154.8 & NA & $62.8(34.3)$ & NA & NA & $9.1(2.8)$ & NA & NA & NA & NA & $83.8(24.4)$ \\
\hline $\begin{array}{l}\text { Smolen et al. } 2009 \text { [28] } \\
\text { MTX-experienced }\end{array}$ & $\operatorname{MTX}(n=127)$ & 84 & $52(12)$ & 78.2 & $67.2(46.8)$ & 1.4 & 40.8 & $1.6(0.6)$ & $6.8(0.9)$ & $21.9(9.7)$ & $30.4(13.4)$ & $46.5(58.6)$ & $23.4(27.7)$ & $23.1(32.1)$ & NA \\
\hline $\begin{array}{l}\text { van der Heijde et al. } \\
2006 \text { [29] } \\
\text { MTX-experienced }\end{array}$ & MTX $(n=228)$ & 76 & 52 & NA & NA & 2.6 & NA & 1.7 & NA & 22.7 & 33.2 & 35.5 & NA & NA & NA \\
\hline $\begin{array}{l}\text { van Holten et al. } 2005 \text { [30] } \\
\text { MTX-experienced }\end{array}$ & $\operatorname{MTX}(n=73)$ & 66 & $54(12)$ & 71.2 & $49.2(28.8)$ & NA & NA & NA & NA & NA & NA & NA & NA & NA & NA \\
\hline \multirow{3}{*}{$\begin{array}{l}\text { Wick et al. } 2005 \text { [31] } \\
\text { DMARD-experienced }\end{array}$} & $\operatorname{MTX}(n=56)$ & 71 & NA & NA & $6.8(3.9)$ & $3.2(4.0)$ & $29.5(20.3)$ & $1.1(0.6)$ & $5.4(1.1)$ & $11(5.6)$ & $9.8(6.2)$ & NA & NA & NA & $15.6(9.6)$ \\
\hline & $\operatorname{SSZ}(n=55)$ & 56 & NA & NA & $6.5(3.6)$ & $1.9(18.0)$ & $28.2(23.1)$ & $0.8(0.5)$ & $4.8(1.1)$ & $10.3(5.8)$ & $7.5(6.0)$ & NA & NA & NA & $12.9(9.4)$ \\
\hline & Gold ( $n=19)$ & 63 & NA & NA & $7.0(4.4)$ & $1.1(9.0)$ & $16.3(9.6)$ & $0.8(0.5)$ & $4.1(0.9)$ & $4.5(3.5)$ & $4.6(3.7)$ & NA & NA & NA & $9.6(7.0)$ \\
\hline \multicolumn{16}{|l|}{ Non-DMARD-IR population } \\
\hline $\begin{array}{l}\text { Bathon et al. } 2000 \text { [32] } \\
\text { DMARD-experienced }\end{array}$ & $\operatorname{MTX}(n=217)$ & 75 & $49(13)$ & 89.0 & $12.0(11.0)$ & $3.7(4.4)$ & NA & NA & NA & $24(11.9)$ & $30.0(16.1)$ & $12.9(13.8)$ & $5.4(6.1)$ & $7.5(9.2)$ & NA \\
\hline $\begin{array}{l}\text { Boiardi et al. } 1999 \text { [33] } \\
\text { DMARD-experienced }\end{array}$ & $\operatorname{MTX}(n=20)$ & 90 & $64(13)$ & 50.0 & $69.0(90.0)$ & $2.5(2.1)$ & $39.0(22.0)$ & NA & NA & $8.9(3.8)$ & NA & NA & NA & NA & $39.4(34.6)$ \\
\hline $\begin{array}{l}\text { Breedveld et al. } 2006 \text { [34] } \\
\text { DMARD-naïve }\end{array}$ & $\operatorname{MTX}(n=257)$ & 74 & $52(13)$ & NA & $9.6(10.8)$ & $4.0(4.0)$ & NA & $1.5(0.6)$ & $6.3(0.9)$ & $22.1(11.7)$ & $32.3(14.3)$ & $21.9(22.2)$ & $8.2(10.7)$ & $13.6(13.6)$ & NA \\
\hline \multirow{2}{*}{$\begin{array}{l}\text { Choy et al. } 2008 \text { [35] } \\
\text { Early RA; DMARD- } \\
\text { experienced }\end{array}$} & $\operatorname{MTX}(n=117)$ & 67 & $54(21-89)^{b}$ & 66.0 & $2.7(3.8)$ & NA & NA & $1.5(0.7)$ & $5.8(1.2)$ & NA & NA & NA & NA & NA & $7^{\mathrm{a}}(3-15)^{\mathrm{b}}$ \\
\hline & $\operatorname{MTX}(n=115)$ & 78 & $54(27-84)^{b}$ & 66.0 & $5.1(5.8)$ & NA & NA & $1.6(0.7)$ & $5.8(1.4)$ & NA & NA & NA & NA & NA & $6^{a}(2-20)^{b}$ \\
\hline
\end{tabular}


Table 2 Patient characteristics at baseline (Continued)

\begin{tabular}{|c|c|c|c|c|c|c|c|c|c|c|c|c|c|c|c|}
\hline \multirow{2}{*}{$\begin{array}{l}\text { Cohen et al. } 2001 \text { [36] } \\
\text { MTX-naïve }\end{array}$} & $\operatorname{LEF}(n=190)$ & 73 & 54 & NA & 82.8 & $2.2(2.8)$ & $38.3(26.0)$ & $0.7(0.5)$ & NA & $13.3(6.3)$ & $13.4(5.6)$ & $23.8(38.5)$ & $13.5(17.2)$ & $10.3(25.6)$ & NA \\
\hline & $\operatorname{MTX}(n=190)$ & 74 & 53 & NA & 78.0 & $2.0(1.9)$ & $35.9(25.7)$ & $0.7(0.5)$ & NA & $13(5.4)$ & $14.3(6.5)$ & $25.1(42.3)$ & $14.5(21.7)$ & $10.6(22.9)$ & NA \\
\hline $\begin{array}{l}\text { Cohen et al. } 2008 \text { [37] } \\
\text { MTX-experienced }\end{array}$ & $\operatorname{DMARD}(n=75)$ & 83 & $57(11)$ & 78.0 & $116.4(97.2)$ & NA & NA & NA & NA & NA & NA & $29.9(34.7)$ & $13.3(18.9)$ & $16.6(17.2)$ & NA \\
\hline \multirow{2}{*}{$\begin{array}{l}\text { Emery et al. } 2000 \text { [38] } \\
\text { DMARD-experienced }\end{array}$} & $\operatorname{LEF}(n=501)$ & 71 & $58(10)$ & NA & $44.4(38.4)$ & NA & NA & NA & NA & $15.8(6.0)$ & $17.2(6.8)$ & NA & NA & NA & $1.3(0.5)$ \\
\hline & MTX $(n=498)$ & 71 & $58(11)$ & NA & $45.6(42.0)$ & NA & NA & NA & NA & $16.5(5.9)$ & $17.7(6.7)$ & NA & NA & NA & $1.3(0.5)$ \\
\hline $\begin{array}{l}\text { Emery et al. } 2008 \text { [39] } \\
\text { MTX-naïve }\end{array}$ & $\operatorname{MTX}(n=263)$ & 73 & $52(1)$ & NA & $9.3(0.4)$ & $3.7(3.4)$ & $49.3(24.1)$ & $1.6(0.7)$ & $6.5(1.0)$ & $17.6(10.0)$ & $24.8(14.5)$ & NA & NA & NA & NA \\
\hline $\begin{array}{l}\text { Emery et al. } 2009 \text { [40] } \\
\text { MTX-naive }\end{array}$ & MTX $(n=257)$ & 74 & $52(13)$ & 84.0 & $10.1(10.7)$ & $4.0(4.0)$ & NA & $1.5(0.7)$ & $6.3(0.9)$ & $22.1(11.7)$ & $32.3(14.3)$ & $21.9(22.2)$ & $8.2(10.7)$ & $13.6(13.5)$ & NA \\
\hline \multirow{2}{*}{$\begin{array}{l}\text { Ferraccioli et al. } 1996 \text { [41] } \\
\text { DMARD-naïve }\end{array}$} & $\operatorname{CSA}(n=141)$ & 77 & $48(12)$ & NA & $16.8(14.4)$ & $8.3(16.7)$ & $46.9(25.2)$ & NA & NA & $14.9(7.5)$ & $23.8(9.6)$ & NA & NA & NA & $25.0(23.0)$ \\
\hline & $\begin{array}{l}\text { Anti-malarial/ } \\
\text { Gold/D-penicill- } \\
\text { amine/SSZ } \\
(n=143)\end{array}$ & 78 & $51(12)$ & NA & $15.6(13.2)$ & $8.5(17.5)$ & $47.5(30.8)$ & NA & NA & $14.7(7.8)$ & $23.2(9.8)$ & NA & NA & NA & $23.8(24.1)$ \\
\hline \multirow{2}{*}{$\begin{array}{l}\text { Ferraccioli et al. } 1997 \text { [42] } \\
\text { DMARD-naïve }\end{array}$} & $\operatorname{CSA}(n=167)$ & 78 & $48(12)$ & NA & 16.8 (14.4) & $7.7(15.5)$ & $48.5(26.2)$ & NA & NA & $15.2(7.7)$ & $23.8(9.4)$ & NA & NA & NA & $26.0(21.8)$ \\
\hline & $\begin{array}{l}\text { Anti-malarial/ } \\
\text { Gold/D-penicill- } \\
\text { amine/SSZ } \\
(n=173)\end{array}$ & 79 & $50(12)$ & NA & $15.6(14.4)$ & $8.7(18.0)$ & $47.6(30.7)$ & NA & NA & $15.1(7.7)$ & $23.9(9.7)$ & NA & NA & NA & $25.0(22.2)$ \\
\hline $\begin{array}{l}\text { Goldbach-Mansky et al. } \\
2009 \text { [43] } \\
\text { MTX-experienced }\end{array}$ & $\operatorname{SSZ}(n=61)$ & 87 & $52(12)$ & NA & NA & $2.5(2.9)$ & $51.0(23.0)$ & NA & $7.0(1.0)$ & $22(13.0)$ & $33.0(17.0)$ & $21.4(31.2)$ & 13.3 & $8.1(0.4)$ & NA \\
\hline $\begin{array}{l}\text { Hannonen et al. } 1993 \text { [44] } \\
\text { DMARD-naïve }\end{array}$ & $\operatorname{SSZ}(n=38)$ & 61 & $52(22-78)^{b}$ & 65.8 & 4.7 & $2.7(3.0)$ & $37.7(21.3)$ & NA & NA & $6.8(3.3)$ & NA & $1.9(0-14.0)^{b}$ & $1.1(0-8.0)^{b}$ & $0.8(0-6.0)^{b}$ & NA \\
\hline $\begin{array}{l}\text { Hetland et al. } 2006 \text { [45] } \\
\text { Early RA; DMARD-naive }\end{array}$ & $\operatorname{MTX}(n=80)$ & 70 & $51^{a}(39.5-62.5)^{c}$ & 59.0 & 46.8 & $1.9^{\mathrm{a}}$ & $27^{\mathrm{a}}$ & $0.9^{\mathrm{a}}$ & $5.5(1.3)$ & $11^{a}(6-15)^{c}$ & $14^{a}(8-20)^{c}$ & NA & NA & NA & $4.6(7.4)$ \\
\hline $\begin{array}{l}\text { Hetland et al. } 2008 \text { [46] } \\
\text { Early RA; MTX-experienced }\end{array}$ & $\operatorname{MTX}(n=80)$ & NA & NA & NA & NA & $1.9^{\mathrm{a}}$ & $27^{\mathrm{a}}$ & $0.9^{\mathrm{a}}$ & $5.7^{\mathrm{a}}$ & $11^{a}(6-15)^{c}$ & $14^{a}(8-20)^{c}$ & $4.8(5.9)$ & $1.7(3.8)$ & $3.1(4.1)$ & NA \\
\hline $\begin{array}{l}\text { Landewé et al. } 2002 \text { [47] } \\
\text { DMARD-naïve }\end{array}$ & $\operatorname{SSZ}(n=74)$ & 53 & NA & 71.0 & 3.6 & NA & NA & $1.4(0.7)$ & $6.1(1.1)$ & NA & NA & $5^{a}(1-10)^{c}$ & NA & NA & NA \\
\hline \multirow{2}{*}{$\begin{array}{l}\text { Larsen et al. } 2001 \text { [48] } \\
\text { DMARD-experienced }\end{array}$} & $\operatorname{LEF}(n=133)$ & 76 & $58(11)$ & NA & $96.0(108.0)$ & NA & NA & NA & NA & NA & NA & NA & NA & NA & $1.5(0.7)$ \\
\hline & $\operatorname{SSZ}(n=133)$ & 69 & $59(11)$ & NA & $84.0(120.0)$ & NA & NA & NA & NA & NA & NA & NA & NA & NA & $1.4(0.7)$ \\
\hline $\begin{array}{l}\text { Maravic et al. } 1999 \text { [49] } \\
\text { MTX-naïve }\end{array}$ & $\operatorname{MTX}(n=29)$ & 72 & 49 (15) & 37.9 & 6.6 & $1.9^{\mathrm{a}}$ & $36^{\mathrm{a}}$ & NA & NA & $12^{\mathrm{a}}(3-27)^{\mathrm{b}}$ & NA & 4.4 & NA & NA & $15.8(15.9)$ \\
\hline \multirow{2}{*}{$\begin{array}{l}\text { Marchesoni et al. } 2002 \text { [50] } \\
\text { DMARD-naïve }\end{array}$} & $\operatorname{CSA}(n=22)$ & NA & $50(12)$ & NA & $9.6(6.0)$ & $0.8(1.1)$ & $29.2(17.4)$ & $0.5(0.5)$ & $3.2(1.2)$ & $3.2(4.0)$ & $4.3(5.2)$ & NA & NA & NA & $5.1(7.0)$ \\
\hline & $\operatorname{MTX}(n=27)$ & NA & $50(12)$ & NA & $9.6(6.0)$ & $0.6(1.3)$ & $29.4(13.2)$ & $0.4(0.5)$ & $3.5(1.0)$ & $3.1(4.6)$ & $4.8(5.7)$ & NA & NA & NA & $5.0(8.8)$ \\
\hline \multirow{2}{*}{$\begin{array}{l}\text { Peltomaa et al. } 1995 \text { [51] } \\
\text { DMARD-naïve }\end{array}$} & Gold $(n=70)$ & 77 & 45 & NA & 7.7 & 2.7 & 35.0 & NA & NA & NA & NA & NA & NA & NA & $5.5(0-22)^{b}$ \\
\hline & SSZ $(n=58)$ & 71 & 49 & NA & 5.8 & 2.8 & 41.0 & NA & NA & NA & NA & NA & NA & NA & $8.9(0-34)^{b}$ \\
\hline $\begin{array}{l}\text { Sanmarti et al. } 2003 \text { [52] } \\
\text { DMARD-naïve }\end{array}$ & $\begin{array}{l}\text { Gold/MTX } \\
(n=60)\end{array}$ & 78 & $52(16)$ & 78.3 & $9.5(6.5)$ & $2.9(3.1)$ & $45.3(27.7)$ & $1.0(0.5)$ & $5.8(0.8)$ & $8.0(4.1)$ & $10.2(5.5)$ & NA & NA & NA & $1.9(3.3)$ \\
\hline
\end{tabular}


Table 2 Patient characteristics at baseline (Continued)

\begin{tabular}{|c|c|c|c|c|c|c|c|c|c|c|c|c|c|c|c|}
\hline $\begin{array}{l}\text { Sarzi-Puttini et al. } 2005 \text { [53] } \\
\text { DMARD-naïve }\end{array}$ & $\operatorname{CSA}(n=36)$ & 78 & $51(10)$ & 66.7 & $14.8(8.4)$ & $3.0(1.8)$ & $45.0(20.0)$ & $1.3(0.6)$ & NA & $12.2(5.6)$ & $15.31(6.41)$ & NA & NA & NA & $14.5(10.0)$ \\
\hline \multirow{2}{*}{$\begin{array}{l}\text { Scott et al. } 1985 \text { [54] } \\
\text { DMARD-naîve }\end{array}$} & DMARD $(n=56)$ & 55 & 56 & NA & NA & 4.9 & 56.5 & NA & NA & NA & NA & NA & NA & NA & $52.0(27.7)$ \\
\hline & NSAID $(n=15)$ & 60 & 52 & NA & NA & 1.9 & 22.6 & NA & NA & NA & NA & NA & NA & NA & $41.3(34.5)$ \\
\hline $\begin{array}{l}\text { Scott et al. } 1989 \text { [55] } \\
\text { DMARD-naïve }\end{array}$ & Gold $(n=49)$ & 61 & $55(3)$ & NA & 24.0 & $0.5(1.1)$ & $57.0(7.0)$ & NA & NA & NA & NA & NA & NA & NA & $33.9(9.8)$ \\
\hline \multirow[t]{2}{*}{$\begin{array}{l}\text { Scott et al. } 1990 \text { [56] } \\
\text { DMARD-naîve }\end{array}$} & $\begin{array}{l}\text { D-penicill-amine } \\
(n=20)\end{array}$ & NA & NA & NA & NA & 6.0 & NA & NA & NA & NA & NA & NA & NA & NA & 25.6 \\
\hline & $\mathrm{HCQ}(n=23)$ & NA & NA & NA & NA & 6.2 & NA & NA & NA & NA & NA & NA & NA & NA & 25.5 \\
\hline \multirow{2}{*}{$\begin{array}{l}\text { Smith et al. } 1982 \text { [57] } \\
\text { DMARD-naïve }\end{array}$} & Gold $(n=26)$ & 69 & 45 & 80.8 & 90.0 & NA & 89.8 & NA & NA & NA & NA & NA & NA & NA & $54.2(6.0)$ \\
\hline & Gold $(n=26)$ & 62 & 46 & 65.4 & 76.8 & NA & 83.4 & NA & NA & NA & NA & NA & NA & NA & $47.4(5.0)$ \\
\hline $\begin{array}{l}\text { St Clair et al. } 2004 \text { [58] } \\
\text { DMARD-experienced }\end{array}$ & $\operatorname{MTX}(n=282)$ & 75 & $50(13)$ & 71.0 & $10.8(8.4)$ & $2.6(2.9)$ & $43.0(28.0)$ & $1.5(0.6)$ & $6.7(1.0)$ & $22.0(11.0)$ & $34(15.0)$ & $11.3(15.9)$ & $3.0(4.8)$ & $8.3(12.3)$ & NA \\
\hline \multirow{2}{*}{$\begin{array}{l}\text { Strand et al. } 1999 \text { [59] } \\
\text { MTX-naiive }\end{array}$} & $\operatorname{LEF}(n=182)$ & 73 & $54(12)$ & 64.8 & $84.0(103.2)$ & $2.1(2.5)$ & $38.4(26.8)$ & $0.8(0.6)$ & NA & $13.7(6.0)$ & $15.5(6.4)$ & $23.1(34.0)$ & $14.2(18.9)$ & $9.0(19.6)$ & NA \\
\hline & $\operatorname{MTX}(n=182)$ & 75 & $53(12)$ & 59.4 & 78.0 & $1.9(1.9)$ & $33.8(25.4)$ & $0.8(0.5)$ & NA & $13.0(5.7)$ & $15.8(6.9)$ & $22.8(39.0)$ & $14.7(23.3)$ & $8.1(18.4)$ & NA \\
\hline $\begin{array}{l}\text { Svensson et al. } 2003 \text { [60] } \\
\text { Early RA; DMARD- } \\
\text { experienced }\end{array}$ & $\operatorname{MTX}(n=50)$ & 58 & 52 & 62.0 & 9.0 & 5.4 & 49.9 & 1.2 & NA & 19.8 & NA & NA & NA & NA & $7.5(10.8)$ \\
\hline \multirow{2}{*}{$\begin{array}{l}\text { Svensson et al. } 2005 \text { [61] } \\
\text { DMARD-naïve }\end{array}$} & $\operatorname{DMARD}(n=119)$ & 65 & $51(14)$ & 66.0 & $6.5(3.5)$ & 2.2 & NA & $1.0(0.6)$ & $5.3(1.1)$ & NA & NA & $4.1(9.2)$ & $2.2(4.6)$ & $1.9(5)$ & NA \\
\hline & $\operatorname{DMARD}(n=131)$ & 63 & $59(13)$ & 66.0 & $5.8(2.9)$ & 2.2 & NA & $1.0(0.7)$ & $5.4(1.0)$ & NA & NA & $4.8(9.6)$ & $2.9(6.4)$ & $1.9(4)$ & NA \\
\hline \multirow{2}{*}{$\begin{array}{l}\text { Van Riel et al. } 1986 \text { [62] } \\
\text { DMARD-naïve }\end{array}$} & Gold $(n=22)$ & 77 & $50(12)$ & NA & $37.2(38.4)$ & NA & $56.0(36.0)$ & NA & NA & NA & NA & NA & NA & NA & $30.9(12.5)$ \\
\hline & Gold $(n=18)$ & 72 & $55(8)$ & NA & $51.6(52.8)$ & NA & $53.0(37.0)$ & NA & NA & NA & NA & NA & NA & NA & $27.6(12.5)$ \\
\hline \multirow[t]{2}{*}{$\begin{array}{l}\text { Wassenberg et al. } 2005 \text { [63] } \\
\text { MTX-naïve }\end{array}$} & $\begin{array}{l}\text { Gold }(66 \%) / \mathrm{MTX} \\
(34 \%)(n=80)\end{array}$ & 25 & $53(13)$ & 43.0 & $8.6(6.7)$ & NA & $44.5(24.9)$ & NA & NA & NA & NA & $11.9(2.1)$ & $6.0(1.3)$ & $6.0(1.08)$ & NA \\
\hline & $\begin{array}{l}\text { Gold }(59 \%) / \mathrm{MTX} \\
(41 \%)(n=86)\end{array}$ & 35 & $50(13)$ & 47.0 & $9.3(6.6)$ & NA & $40.1(24.6)$ & NA & NA & NA & NA & $9.4(1.9)$ & $4.5(1.2)$ & $4.7(0.87)$ & NA \\
\hline
\end{tabular}

AZA azathioprine, CRP C-reactive protein, CSA ciclosporin A, DAS-28 disease activity score in 28 joints, DMARD disease-modifying anti-rheumatic drug, DMARD-IR patient population with moderate-to-severe RA with a history of inadequate response to non-biologic DMARDs who are currently treated with one (other) non-biologic DMARD, ES Erosion Subscore of TSS, ESR erythrocyte sedimentation rate, gold gold salts, HAQ Health Assessment Questionnaire, HCQ hydroxychloroquine, ITT intent to treat, JSN Joint Space Narrowing Subscore of TSS, LEF leflunomide, MTX methotrexate, NA not available, non-DMARD-IR patient population with moderate-to-severe RA without a history of inadequate response to a DMARD who are currently receiving palliative care (NSAIDs, analgesics, low-dose glucocorticoids) or are being minimally treated with one non-biologic DMARD, NSAID non-steroidal anti-inflammatory drug, RA rheumatoid arthritis, Ref reference, $R F+$ rheumatoid factor positive, SJC swollen joint count, SSZ sulfasalazine, TJC tender joint count,

TSS modified Total Sharp Score

aMedian. ${ }^{b}$ Range. Interquartile range 


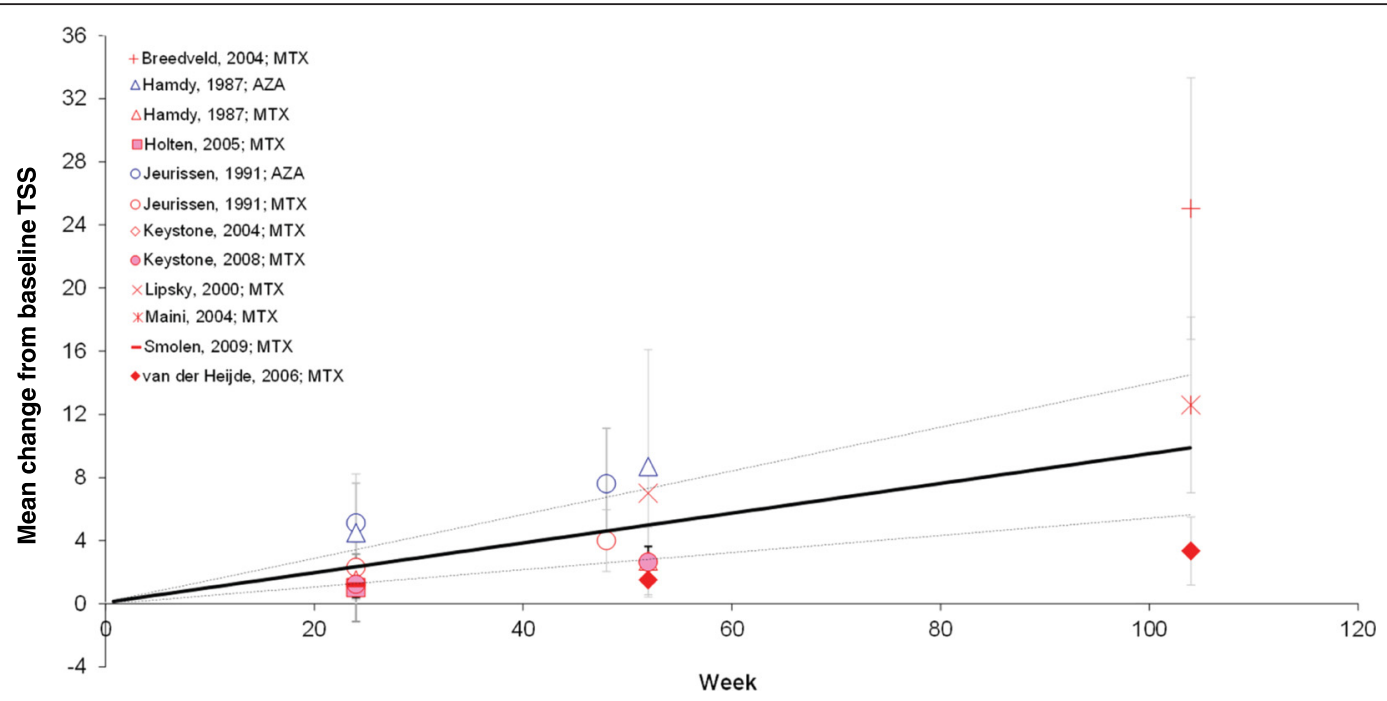

Fig. 2 Mean change from baseline in TSS in the DMARD-IR population. Data are as observed in individual studies and estimated with meta-analysis. Solid line represents the mean estimate for a given treatment arm and the dashed lines show the corresponding $95 \%$ credible interval. AZA: azathioprine; DMARD-IR: patient population with moderate-to-severe rheumatoid arthritis with a history of inadequate response to disease-modifying anti-rheumatic drugs (DMARDs) who are currently treated with one (other) non-biologic DMARD; MTX: methotrexate; TSS: modified Total Sharp Score

biologic DMARD-naïve and non-biologic DMARD-experienced patients without an inadequate response to any DMARD. In the identified studies, the minimally treated DMARD-IR patients were receiving monotherapy with MTX, AZA, SSZ, or gold salts, with most patients receiving MTX. In the included nonDMARD-IR studies, DMARD treatment consisted of MTX, SSZ, LEF, CSA, HCQ, or D-penicillamine. Only one study was identified in which patients were treated with NSAIDs only, but this study was not included in the meta-analysis. For both populations, treatment with one DMARD resulted in deterioration of joint structure over a 2-year period as measured with the TSS, ES, JSN, and Larsen scores. Under the assumption that the minimal clinically important difference is about $1 \%$ of the maximum of the possible TSS and Larson scores, the estimated changes over a 2 year period in terms of TSS can be considered relevant, in particular for the DMARD-IR population [64, 65]. Depending on the time assessed and the measure examined, the rate of deterioration in the DARD-IR population was about 1.5- to 2-times the rate of deterioration in the non-DMARD-IR population. Based on RCT evidence, the rate of deterioration with AZA was greater than with MTX in the DMARD-IR population. For the non-DMARD-IR population, LEF and MTX showed a similar progression over time.

The greater rate of deterioration observed in the DMARD-IR population compared with the nonDMARD-IR population makes sense, given the negative impact a history of non-biologic-DMARD failure should have on the effectiveness of continuation with a non- biologic DMARD. Related underlying causes for the difference in progression rates are possibly differences in disease duration, rheumatoid factor status, and disease activity.

However, it is important to note that for a subset of the identified studies it was not clear whether the patients were exclusively DMARD-IR. These studies were assigned to the non-DMARD-IR group to make sure that the DMARD-IR group was as homogenous as possible. As such, it is possible that the defined non-DMARD-IR population partly consisted of patients who might have a history of failed treatment with a DMARD. This possible misclassification might have overestimated the deterioration in this group, and should be kept in mind when comparing the degree of joint deterioration in DMARD-IR versus non-DMARD-IR populations.

The relevant studies were identified by means of a systematic search of the literature and included both RCTs and observational designs. Given the objective of the meta-analysis, only those arms of the comparative studies were selected in which patients were treated with NSAIDs or a single DMARD (with or without additional NSAIDs or corticosteroid use). Although many RCTs were included, often only one treatment arm (e.g., MTX-only arm from biologic trials in DMARD-IR populations) was used. As such, there was no difference in the way evidence obtained from observational studies and RCTs was handled. RCTs in which different single nonbiologic DMARDs were compared were included and provided the evidence to allow comparisons between DMARDs. Comparative analyses were only possible for 
Table 3 Mean change from baseline in TSS and subscores in the DMARD-IR population as estimated with meta-analysis

\begin{tabular}{|c|c|c|c|c|c|c|c|c|c|c|c|c|}
\hline & \multicolumn{3}{|c|}{ Week 12} & \multicolumn{3}{|c|}{ Week 24} & \multicolumn{3}{|c|}{ Week 52} & \multicolumn{3}{|c|}{ Week 104} \\
\hline & CFB & $95 \% \mathrm{Crl}$ & $\begin{array}{l}\text { Probability of } \\
\text { deterioration } \\
\text { relative to } \\
\text { baseline }\end{array}$ & CFB & $95 \% \mathrm{Crl}$ & $p$-value & CFB & $95 \% \mathrm{Crl}$ & $\begin{array}{l}\text { Probability of } \\
\text { deterioration } \\
\text { relative to } \\
\text { baseline }\end{array}$ & CFB & $95 \% \mathrm{Crl}$ & $\begin{array}{l}\text { Probability of } \\
\text { deterioration } \\
\text { relative to } \\
\text { baseline }\end{array}$ \\
\hline \multicolumn{13}{|l|}{ TSS } \\
\hline DMARDs & 1.14 & $(0.66,1.67)$ & $>0.99$ & 2.27 & $(1.31,3.34)$ & $>0.99$ & 4.92 & $(2.84,7.23)$ & $>0.99$ & 9.84 & $(5.68,14.46)$ & $>0.99$ \\
\hline MTX & 0.97 & $(0.49,1.52)$ & 0.99 & 1.95 & $(0.98,3.05)$ & 0.99 & 4.22 & $(2.13,6.6)$ & 0.99 & 8.44 & $(4.26,13.21)$ & 0.99 \\
\hline$A Z A^{a}$ & 2.16 & $(2.16,3.04)$ & $>0.99$ & 4.32 & $(2.59,6.07)$ & $>0.99$ & 9.35 & $(5.62,13.15)$ & $>0.99$ & 18.70 & $(11.24,26.31)$ & $>0.99$ \\
\hline \multicolumn{13}{|c|}{ Erosion Subscore } \\
\hline DMARDs & 0.51 & $(0.27,0.83)$ & 0.99 & 1.02 & $(0.55,1.66)$ & 0.99 & 2.22 & $(1.19,3.6)$ & 0.99 & 4.43 & $(2.38,7.21)$ & 0.99 \\
\hline MTX & 0.43 & $(0.25,0.69)$ & 0.98 & 0.87 & $(0.49,1.39)$ & 0.98 & 1.88 & $(1.07,3.01)$ & 0.98 & 3.76 & $(2.14,6.01)$ & 0.98 \\
\hline$A Z A^{a}$ & 1.31 & $(1.31,1.98)$ & $>0.99$ & 2.62 & $(1.3,3.96)$ & $>0.99$ & 5.67 & $(2.81,8.57)$ & $>0.99$ & 11.35 & $(5.61,17.14)$ & $>0.99$ \\
\hline \multicolumn{13}{|c|}{ Joint Space Narrowing Subscore } \\
\hline DMARDs & 0.36 & $(0.09,0.67)$ & 0.99 & 0.72 & $(0.18,1.33)$ & 0.99 & 1.57 & $(0.4,2.89)$ & 0.99 & 3.14 & $(0.80,5.78)$ & 0.90 \\
\hline
\end{tabular}

$A Z A$ azathioprine, $\mathrm{Crl}$ credible interval; DMARD disease-modifying anti-rheumatic drug; DMARD-IR patient population with moderate-to-severe rheumatoid arthritis with a history of inadequate response to non-biologic DMARDs who are currently treated with one (other) non-biologic DMARD, CFB change from baseline; MTX methotrexate, $p$-value probability of joint structural deterioration relative to baseline; TSS modified Total Sharp Score ${ }^{a}$ Estimated based on comparative data only, using models for relative treatment effects (see Model 3 and 4 in Additional file 2), which allows comparative interpretation of MTX and AZA findings

AZA versus MTX and for LEF versus MTX. Although RCTs provided comparative data for some other DMARDs, these were not part of a connected network of RCTs and could not, therefore, be used in the planned analyses.

Many of the MTX treatment arms comprising the DMARD-IR population were obtained from RCTs in which a biologic DMARD was evaluated. In the included studies the patients in these MTX arms were not assigned to biologic treatment within the study time horizon; only the MTX dose could be increased in case of non- response. Hence, the observed structural deterioration is a reflection of the limitations of MTX in this population.

The included studies reported joint deterioration at different time points, with outcomes reported up to 2 years of follow-up. With the meta-analysis models used, all the available time points were analyzed simultaneously to estimate a curve reflecting joint structural deterioration over time. It cannot be assumed that extrapolation of these curves beyond this 2-year period is a valid representation of joint structure deterioration over the longer term. The

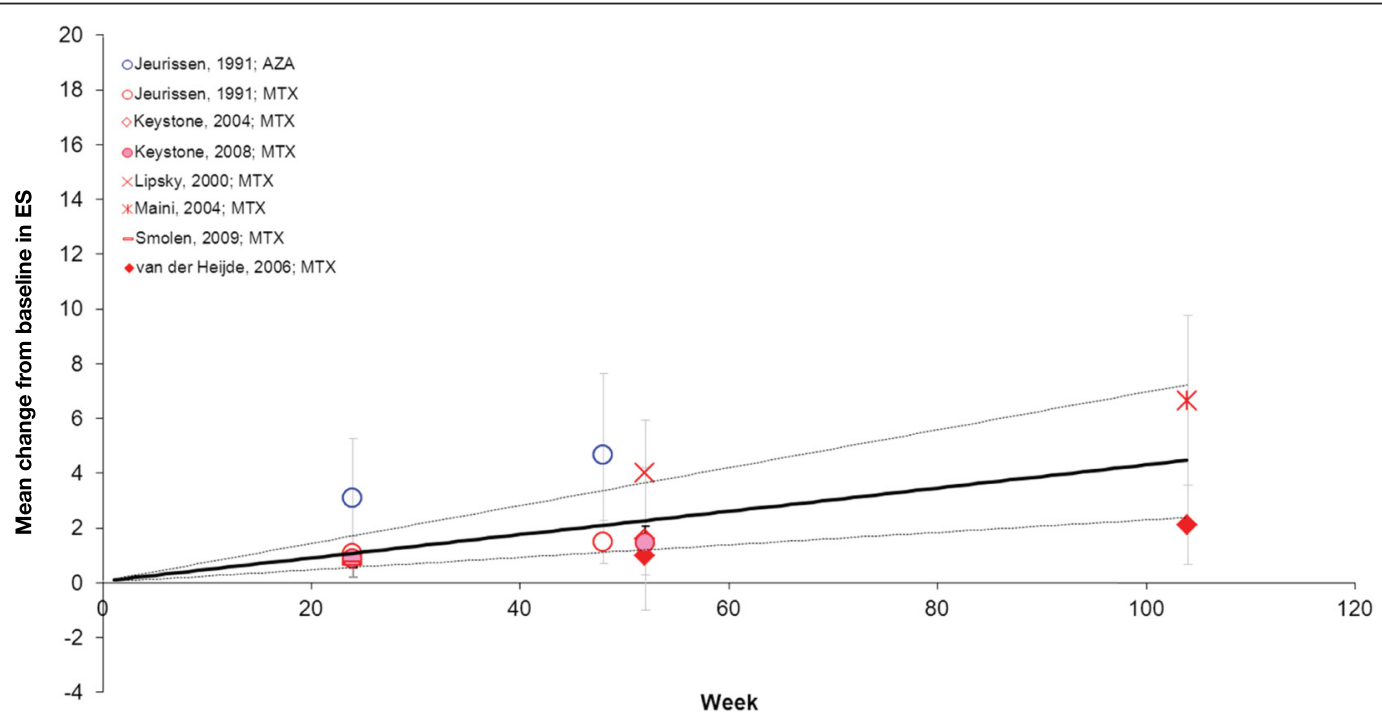

Fig. 3 Mean change from baseline in ES in the DMARD-IR population. Data are as observed in individual studies and estimated with meta-analysis. Solid line represents the mean estimate for a given treatment arm and the dashed lines show the corresponding $95 \%$ credible interval. AZA: azathioprine; DMARD-IR: patient population with moderate-to-severe rheumatoid arthritis with a history of inadequate response to disease-modifying anti-rheumatic drugs (DMARDs) who are currently treated with one (other) non-biologic DMARD; ES: Erosion Subscore; MTX: methotrexate 


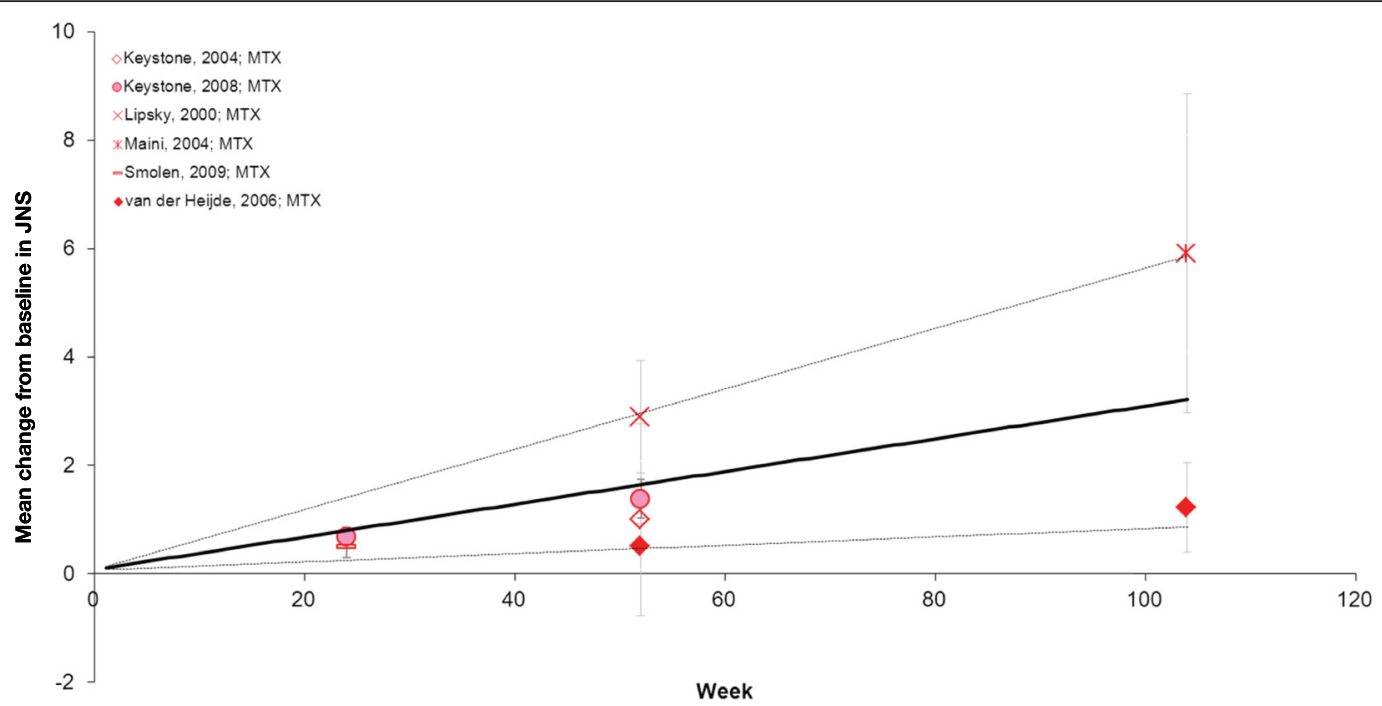

Fig. 4 Mean change from baseline in JSN Subscore in the DMARD-IR population. Data are as observed in individual studies and estimated with meta-analysis. Solid line represents the mean estimate for a given treatment arm and the dashed lines show the corresponding $95 \%$ credible interval. DMARD-IR: patient population with moderate-to-severe rheumatoid arthritis with a history of inadequate response to disease-modifying anti-rheumatic drugs (DMARDs) who are currently treated with one (other) non-biologic DMARD; JSN: Joint Space Narrowing; MTX: methotrexate

vast majority of studies used the modified Sharp score to analyze joint erosion and space narrowing. The modified score includes feet in the radiographic assessment, in addition to the scoring of wrists and hands as with the original Sharp score [6-10]. The study by Hamdy [21] used the earlier version of the Sharp score. Despite the differences in total score, we included the study by Hamdy in the analysis of the DMARD-IR population. We do not expect this variation in total score to be a cause of large between-study heterogeneity in development of TSS over time. In fact the observed TSS reported by Hamdy is very consistent with the other studies included in that analysis (Fig. 2).

The included studies were characterized by variability in patient characteristics, especially among the nonDMARD-IR studies. As a result, heterogeneity in joint structural deterioration over time was observed. In order to capture this heterogeneity, random-effects models were used; however, these models do not explain the heterogeneity. In the future, it will be of interest to evaluate whether

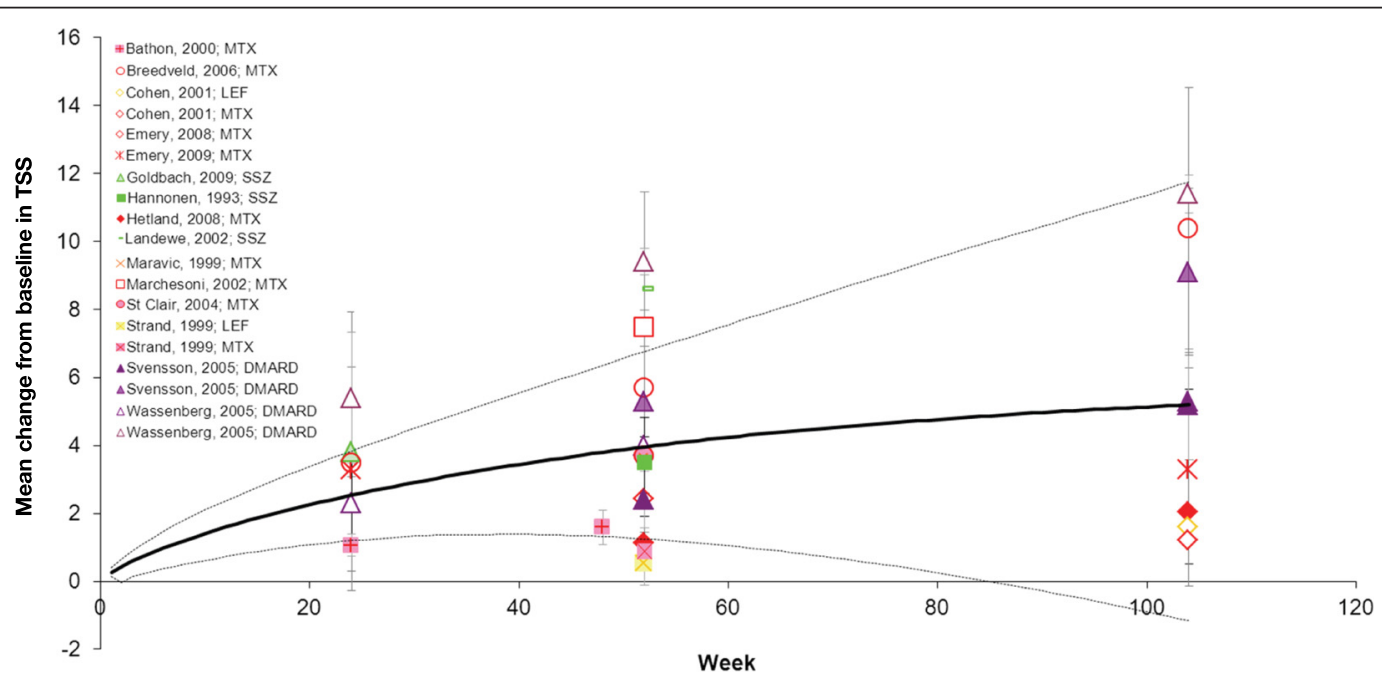

Fig. 5 Mean change from baseline in TSS in the non-DMARD-IR population. Data are as observed in individual studies and estimated with meta-analysis. Solid line represents the mean estimate for a given treatment arm and the dashed lines show the corresponding $95 \%$ credible interval. AZA: azathioprine; DMARD: disease-modifying anti-rheumatic drugs; DMARD-IR: patient population with moderate-to-severe rheumatoid arthritis with a history of inadequate response to DMARDs who are currently treated with one (other) non-biologic DMARD; LEF: leflunomide; MTX: methotrexate; SSZ: sulfasalazine; TSS: modified Total Sharp Score 
Table 4 Mean change from baseline in TSS and subscores plus Larsen Score in the non-DMARD-IR population as estimated with meta-analysis

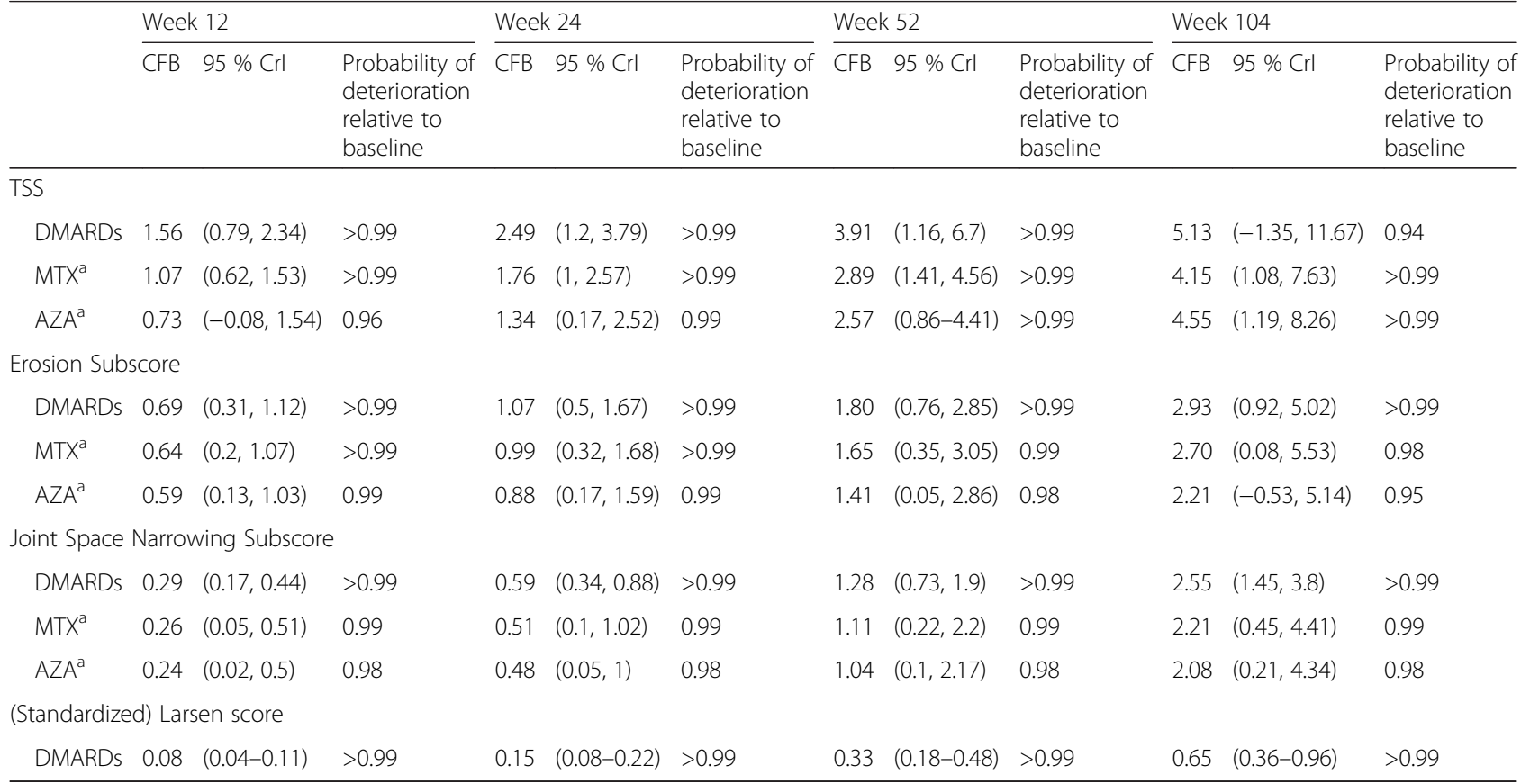

AZA azathioprine, CFB change from baseline, Crl credible interval, DMARD disease-modifying anti-rheumatic drug, MTX methotrexate, non-DMARD-IR patient population with moderate-to-severe rheumatoid arthritis without a history of inadequate response to a DMARD who are currently receiving palliative care (non-steroidal anti-inflammatory drugs, analgesics, low-dose glucocorticoids) or are being minimally treated with one non-biologic DMARD, $p$-value probability of joint structural deterioration relative to baseline, TSS modified Total Sharp Score

aEstimated based on comparative data only, using models for relative treatment effects (see Model 3 and 4 in Additional file 2), which allows comparative interpretation of MTX and AZA findings

certain patient characteristics are associated with differences in joint deterioration. However, meta-regression analysis where study level data is used to evaluate the impact of patient characteristics on outcomes or treatment effects can be prone to ecological bias $[66,67]$. For such an evaluation it is preferred to have access to patient-level data. In this context it would be interesting to evaluate the independent effect of steroid use, disease duration, rheumatoid factor status, and disease activity on joint deterioration, for example.

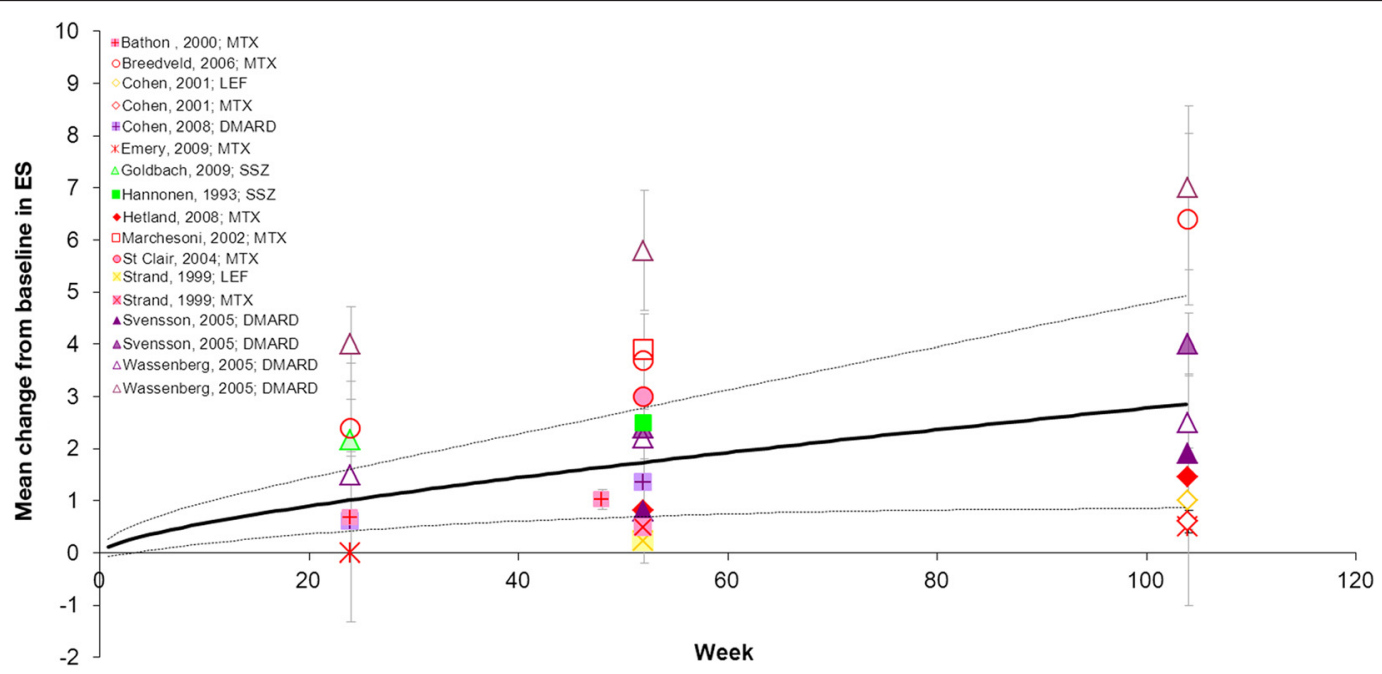

Fig. 6 Mean change from baseline in ES in the non-DMARD-IR population. Data are as observed in individual studies and estimated with meta-analysis. Solid line represents the mean estimate for a given treatment arm and the dashed lines show the corresponding $95 \%$ credible interval. DMARD: diseasemodifying anti-rheumatic drugs; DMARD-IR: patient population with moderate-to-severe rheumatoid arthritis with a history of inadequate response to DMARDs who are currently treated with one (other) non-biologic DMARD; ES: Erosion Subscore; LEF: leflunomide; MTX: methotrexate; SSZ: sulfasalazine 


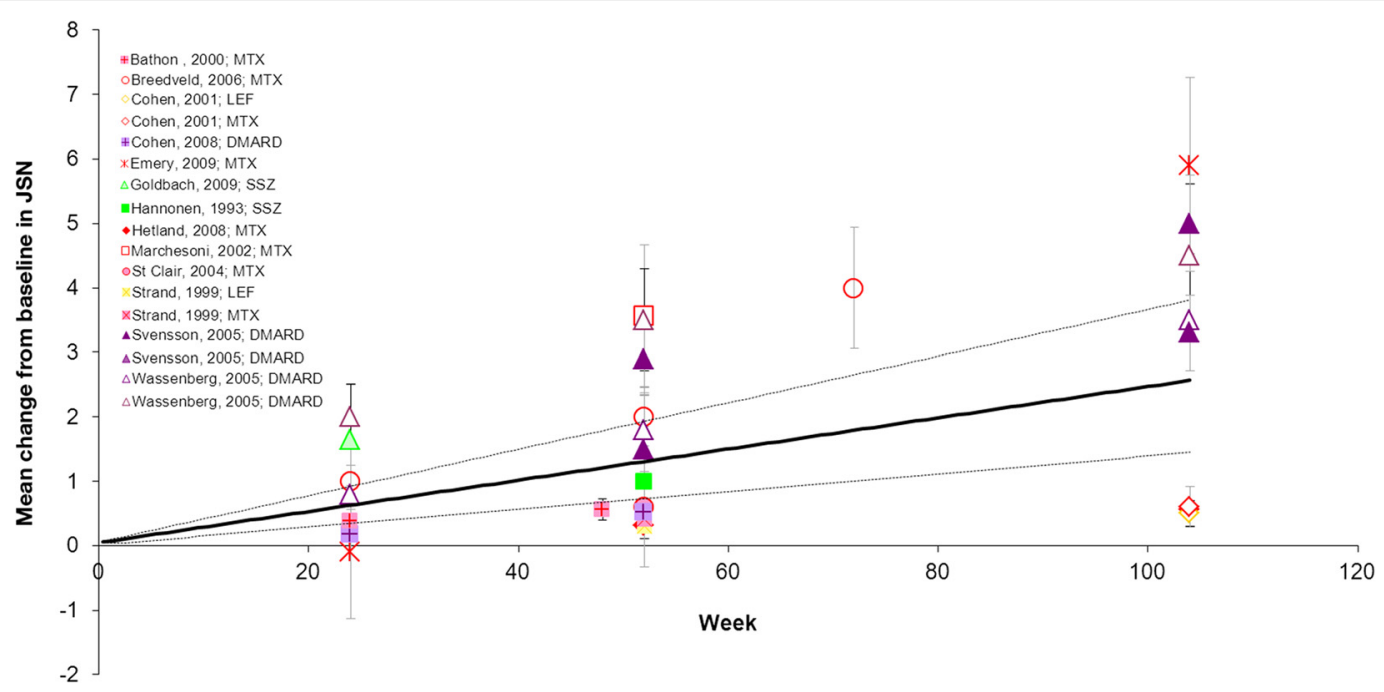

Fig. 7 Mean change from baseline in JSN Subscore in the non-DMARD-IR. Data are as observed in individual studies and estimated with meta-analysis. Solid line represents the mean estimate for a given treatment arm and the dashed lines show the corresponding $95 \%$ credible interval. DMARD: diseasemodifying anti-rheumatic drugs; DMARD-IR: patient population with moderate-to-severe rheumatoid arthritis with a history of inadequate response to DMARDs who are currently treated with one (other) non-biologic DMARD; JSN: Joint Space Narrowing; LEF: leflunomide; MTX: methotrexate; SSZ: sulfasalazine

There is evidence that the patients treated in biologic trials have changed substantially over the past decade. [68]. This is important to consider when using the findings of this meta-analysis to help interpret results of new placebo-controlled trials of biologics. In this context, a limitation of the current analysis is that any potentially relevant studies published after 2009 were not included.

\section{Conclusions}

Based on the currently available published evidence, it can be concluded that minimal treatment of RA with one non-biologic DMARD results in a high risk of deterioration of joint structures among patients who have shown an inadequate response to non-biologic DMARDs, as well as patients that have not (yet) shown an inadequate response. This finding is of relevance when assessing the relative benefit of arresting joint damage with new biologic agents based on findings of placebo-controlled trials in which patients randomized to placebo are 'rescued' with active therapy within 6 months.

\section{Additional files}

Additional file 1: Search strategy. (DOC $23 \mathrm{~kb})$

Additional file 2: Meta analysis models. (DOCX $83 \mathrm{~kb})$

\section{Abbreviations}

AZA: azathioprine; CFB: change from baseline; Crl: credible interval; CRP: C-reactive protein; CSA: ciclosporin A; DIC: deviance information criterion; DMARD: disease-modifying anti-rheumatic drugs; DMARD-IR: inadequate responder to DMARD therapy; ES: Erosion Subscore; ESR: erythrocyte sedimentation rate; HAQ: Health Assessment Questionnaire; HCQ: hydroxychloroquine; JSN: Joint Space Narrowing; LEF: leflunomide;
MTX: methotrexate; NSAID: non-steroidal anti-inflammatory drug; RA: rheumatoid arthritis; RCT: randomized controlled trial; SD: standard deviation; SSZ: sulfasalazine; TSS: Total Sharp Score

\section{Acknowledgements}

Editorial support was provided by Martin Goulding, PhD, at Complete Medical Communications and was funded by Pfizer Inc.

\section{Funding}

This study was sponsored by Pfizer Inc. As specified above, all authors were funded by Pfizer either as consultants or employees and Pfizer were involved in the decision to submit this manuscript for publication.

\section{Availability of data and materials}

All data supporting the findings is contained in the manuscript. Two supplementary files include the literature search strategy and statistical models used for the meta-analysis.

\section{Authors' contributions}

JPJ designed the study, performed the analyses, and co-wrote the manuscript MCV coordinated and helped conduct the systematic literature review and data extraction, performed part of the analyses, and co-wrote the manuscript. JCC made substantial contributions to the analysis and interpretation of data, and revised the article critically for important intellectual content. JDB, SHZ, and GCW made substantial contributions to the study design, interpretation of results, and development of the manuscript. All authors read and approved the final version of the manuscript.

\section{Competing interests}

At the time of performing the analysis presented this manuscript, JPJ and MCV were employees of Mapi and were paid consultants to Pfizer Inc in connection with the manuscript's development. All other authors are employees of Pfizer Inc.

Consent for publication

Not applicable.

Ethics approval and consent to participate

Not applicable as this is a meta-analysis of published data. 


\section{Author details}

${ }^{1}$ Tufts University School of Medicine, Boston, MA, USA. ${ }^{2}$ Formerly at Mapi, Boston, MA, USA. ${ }^{3}$ Pfizer Medicines Development Group, Groton, CT, USA ${ }^{4} 1714$ Stockton Street, 3rd floor, San Francisco, CA 94133, USA.

\section{Received: 25 March 2014 Accepted: 31 May 2016}

\section{Published online: 18 August 2016}

\section{References}

1. American College of Rheumatology Subcommittee on Rheumatoid Arthritis Guidelines. Guidelines for the management of rheumatoid arthritis: 2002 update. Arthritis Rheum. 2002:46(2):328-46.

2. Ledingham J, Deighton C, British Society for Rheumatology Standards, Guidelines and Audit Working Group. Update on the British Society for Rheumatology guidelines for prescribing TNFalpha blockers in adults with rheumatoid arthritis (update of previous guidelines of April 2001). Rheumatology (Oxford). 2005:44(2):157-63.

3. Saag KG, Teng GG, Patkar NM, Anuntiyo J, Finney C, Curtis JR, Paulus HE, Mudano A, Pisu M, Elkins-Melton M, Outman R, Allison JJ, Suarez Almazor M, Bridges Jr SL, Chatham WW, Hochberg M, MacLean C, Mikuls T, Moreland LW, O'Dell J, Turkiewicz AM, Furst DE, American College of Rheumatology. American College of Rheumatology 2008 recommendations for the use of nonbiologic and biologic disease-modifying antirheumatic drugs in rheumatoid arthritis. Arthritis Rheum. 2008:59(6):762-84.

4. Larsen A. A radiological method for grading the severity of rheumatoid arthritis, Academic dissertation. Helsinki: University of Helsinki; 1974.

5. Larsen A, Dale K, Eek M. Radiographic evaluation of rheumatoid arthritis and related conditions by standard reference films. Acta Radiol Diagn (Stockh). 1977:18(4):481-91.

6. Sharp JT, Lidsky MD, Collins LC, Moreland J. Methods of scoring the progression of radiologic changes in rheumatoid arthritis. Correlation of radiologic, clinical and laboratory abnormalities. Arthritis Rheum. 1971;14(6):706-20.

7. Sharp JT, Lidsky MD, Duffy J. Clinical responses during gold therapy for rheumatoid arthritis. Changes in synovitis, radiologically detectable erosive lesions, serum proteins, and serologic abnormalities. Arthritis Rheum. 1982; 25(5):540-9.

8. Sharp JT, Young DY, Bluhm GB, Brook A, Brower AC, Corbett M, Decker JL, Genant HK, Gofton JP, Goodman N, et al. How many joints in the hands and wrists should be included in a score of radiologic abnormalities used to assess rheumatoid arthritis? Arthritis Rheum. 1985;28(12):1326-35.

9. van der Heijde D. How to read radiographs according to the Sharp/van der Heijde method. J Rheumatol. 1999;26(3):743-5.

10. van der Heijde D, Boers M, Lassere M. Methodological issues in radiographic scoring methods in rheumatoid arthritis. J Rheumatol. 1999;26(3):726-30.

11. Moher D, Liberati A, Tetzlaff J, Altman DG, PRISMA Group. Preferred reporting items for systematic reviews and meta-analyses: The PRISMA statement. BMJ. 2009;339:b2535. doi:10.1136/bmj.b2535.

12. Jansen JP, Vieira MC, Cope S. Network meta-analysis of longitudinal data using fractional polynomials. Stat Med. 2015;34(15):2294-311.

13. Sutton AJ, Abrams KR. Bayesian methods in meta-analysis and evidence synthesis. Stat Methods Med Res. 2001;10(4):277-303.

14. Sauerbrei W, Royston P. Building multivariable prognostic and diagnostic models: transformation of the predictors by using fractional polynomials. J R Stat Soc Ser A Stat Soc. 1999;162(1):71-94.

15. Royston P, Altman DG. Regression using fractional polynomials of continuous covariates: parsimonious parametric modelling. Appl Statist. 1994;43(3):429-67.

16. Bagnardi V, Zambon A, Quatto P, Corrao G. Flexible meta-regression functions for modeling aggregate dose response data, with an application to alcohol and mortality. Am J Epidemiol. 2004;159(11):1077-86.

17. Dempster AP. The direct use of likelihood for significance testing. Stat Comput. 1997;7(4):247-52.

18. Spiegelhalter DJ, Best NG, Carlin BP, van der Linde A. Bayesian measures of model complexity and fit. J R Stat Soc Ser B Stat Methodol. 2002;64(4):583-639.

19. Spiegelhalter DJ, Thomas A, Best NG, Lunn D. WinBUGS user manual version 14. Cambridge: MRC Biostatistics Unit; 2002.

20. Breedveld FC, Emery P, Keystone E, Patel K, Furst DE, Kalden JR, St Clair EW, Weisman M, Smolen J, Lipsky PE, Maini RN. Infliximab in active early rheumatoid arthritis. Ann Rheum Dis. 2004;63(2):149-55.

21. Hamdy H, McKendry RJ, Mierins E, Liver JA. Low-dose methotrexate compared with azathioprine in the treatment of rheumatoid arthritis. A twenty-four-week controlled clinical trial. Arthritis Rheum. 1987;30(4):361-8.
22. Jeurissen $M E$, Boerbooms $A M$, van de Putte $L B$, Doesburg $W H$, Lemmens $A$. Influence of methotrexate and azathioprine on radiologic progression in rheumatoid arthritis. A randomized, double-blind study. Ann Intern Med. 1991;114(12):999-1004.

23. Keystone EC, Kavanaugh AF, Sharp JT, Tannenbaum H, Hua Y, Teoh LS, Fischkoff SA, Chartash EK. Radiographic, clinical, and functional outcomes of treatment with adalimumab a human anti-tumor necrosis factor monoclonal antibody in patients with active rheumatoid arthritis receiving concomitant methotrexate therapy: a randomized, placebo-controlled, 52week trial. Arthritis Rheum. 2004;50(5):1400-11.

24. Keystone E, Heijde D, Mason Jr D, Landewé R, Vollenhoven RV, Combe B, Emery P, Strand V, Mease P, Desai C, Pavelka K. Certolizumab pegol plus methotrexate is significantly more effective than placebo plus methotrexate in active rheumatoid arthritis: findings of a fifty-two-week, phase III, multicenter, randomized, double-blind, placebo-controlled, parallel-group study. Arthritis Rheum. 2008;58(11):3319-29.

25. Lipsky PE, van der Heijde DM, St Clair EW, Furst DE, Breedveld FC, Kalden JR, Smolen JS, Weisman M, Emery P, Feldmann M, Harriman GR, Maini RN, AntiTumor Necrosis Factor Trial in Rheumatoid Arthritis with Concomitant Therapy Study Group. Infliximab and methotrexate in the treatment of rheumatoid arthritis. Anti-tumor necrosis factor trial in rheumatoid arthritis with concomitant therapy study group. N Engl J Med. 2000;343(22):1594-602.

26. Maini RN, Breedveld FC, Kalden JR, Smolen JS, Furst D, Weisman MH, St Clair EW, Keenan GF, van der Heijde D, Marsters PA, Lipsky PE, Anti-Tumor Necrosis Factor Trial in Rheumatoid Arthritis with Concomitant Therapy Study Group. Sustained improvement over two years in physical function, structural damage, and signs and symptoms among patients with rheumatoid arthritis treated with infliximab and methotrexate. Arthritis Rheum. 2004:50(4):1051-65.

27. Sany J, Kaliski S, Couret M, Cuchacovich M, Daures JP. Radiologic progression during intramuscular methotrexate treatment of rheumatoid arthritis. J Rheumatol. 1990;17(12):1636-41

28. Smolen J, Landewé RB, Mease P, Brzezicki J, Mason D, Luijtens K, van Vollenhoven RF, Kavanaugh A, Schiff M, Burmester GR, Strand V, Vencovsky J, van der Heijde D. Efficacy and safety of certolizumab pegol plus methotrexate in active rheumatoid arthritis: the RAPID 2 study. A randomised controlled trial. Ann Rheum Dis. 2009;68(6):797-804.

29. van der Heijde D, Klareskog L, Rodriguez-Valverde V, Codreanu C, Bolosiu H, Melo-Gomes J, Tornero-Molina J, Wajdula J, Pedersen R, Fatenejad S, TEMPO Study Investigators. Comparison of etanercept and methotrexate, alone and combined, in the treatment of rheumatoid arthritis: two-year clinical and radiographic results from the TEMPO study, a double-blind, randomized trial. Arthritis Rheum. 2006;54(4):1063-74.

30. van Holten J, Pavelka K, Vencovsky J, Stahl H, Rozman B, Genovese M, Kivitz AJ, Alvaro J, Nuki G, Furst DE, Herrero-Beaumont G, McInnes IB, Musikic P, Tak PP. A multicentre, randomised, double blind, placebo controlled phase II study of subcutaneous interferon beta-1a in the treatment of patients with active rheumatoid arthritis. Ann Rheum Dis. 2005:64(1):64-9.

31. Wick MC, Lindblad S, Weiss RJ, Klareskog L, van Vollenhoven RF. Estimated prediagnosis radiological progression: an important tool for studying the effects of early disease modifying antirheumatic drug treatment in rheumatoid arthritis. Ann Rheum Dis. 2005;64(1):134-7.

32. Bathon JM, Martin RW, Fleischmann RM, Tesser JR, Schiff MH, Keystone EC, Genovese MC, Wasko MC, Moreland LW, Weaver AL, Markenson J, Finck BK. A comparison of etanercept and methotrexate in patients with early rheumatoid arthritis. N Engl J Med. 2000;343(22):1586-93.

33. Boiardi L, Macchioni P, Meliconi R, Pulsatelli L, Facchini A, Salvarani C. Relationship between serum RANTES levels and radiological progression in rheumatoid arthritis patients treated with methotrexate. Clin Exp Rheumatol. 1999;17(4):419-25.

34. Breedveld FC, Weisman MH, Kavanaugh AF, Cohen SB, Pavelka K, van Vollenhoven R, Sharp J, Perez JL, Spencer-Green GT. The PREMIER study: a multicenter, randomized, double-blind clinical trial of combination therapy with adalimumab plus methotrexate versus methotrexate alone or adalimumab alone in patients with early, aggressive rheumatoid arthritis who had not had previous methotrexate treatment. Arthritis Rheum. 2006;54(1):26-37.

35. Choy EH, Smith CM, Farewell V, Walker D, Hassell A, Chau L, Scott DL, CARDERA (Combination Anti-Rheumatic Drugs in Early Rheumatoid Arhritis) Trial Group. Factorial randomised controlled trial of glucocorticoids and combination disease modifying drugs in early rheumatoid arthritis. Ann Rheum Dis. 2008;67(5):656-63.

36. Cohen S, Cannon GW, Schiff M, Weaver A, Fox R, Olsen N, Furst D, Sharp J, Moreland L, Caldwell J, Kaine J, Strand V. Two-year, blinded, randomized, 
controlled trial of treatment of active rheumatoid arthritis with leflunomide compared with methotrexate. Utilization of Leflunomide in the Treatment of Rheumatoid Arthritis Trial Investigator Group. Arthritis Rheum. 2001;44(9): 1984-92.

37. Cohen SB, Dore RK, Lane NE, Ory PA, Peterfy CG, Sharp JT, van der Heijde D, Zhou L, Tsuji W, Newmark R, Denosumab Rheumatoid Arthritis Study Group. Denosumab treatment effects on structural damage, bone mineral density, and bone turnover in rheumatoid arthritis: a twelve-month, multicenter, randomized, double-blind, placebo-controlled, phase II clinical trial. Arthritis Rheum. 2008:58(5):1299-309.

38. Emery P, Breedveld FC, Lemmel EM, Kaltwasser JP, Dawes PT, Gömör B, Van Den Bosch F, Nordström D, Bjorneboe O, Dahl R, Horslev-Petersen K, Rodriguez De La Serna A, Molloy M, Tikly M, Oed C, Rosenburg R, LoewFriedrich I. A comparison of the efficacy and safety of leflunomide and methotrexate for the treatment of rheumatoid arthritis. Rheumatology (Oxford). 2000;39(6):655-65.

39. Emery $P$, Breedveld FC, Hall S, Durez $P$, Chang DJ, Robertson D, Emery $P$, Breedveld FC, Hall S, Durez P, Chang DJ, Robertson D, Singh A, Pedersen $\mathrm{RD}$, Koenig AS, Freundlich B. Comparison of methotrexate monotherapy with a combination of methotrexate and etanercept in active, early, moderate to severe rheumatoid arthritis (COMET): a randomised, doubleblind, parallel treatment trial. Lancet. 2008;372(9636):375-82.

40. Emery P, Genovese MC, van Vollenhoven R, Sharp JT, Patra K, Sasso EH. Less radiographic progression with adalimumab plus methotrexate versus methotrexate monotherapy across the spectrum of clinical response in early rheumatoid arthritis. J Rheumatol. 2009;36(7):1429-41.

41. Ferraccioli GF, Della Casa-Alberghi O, Marubini E, Priolo F, Mathieu A, Fantini F, Cutolo M, Pasero G. Is the control of disease progression within our grasp? Review of the GRISAR study. Gruppo Reumatologi Italiani Studio Artrite Reumatoide. Br J Rheumatol. 1996;35 Suppl 2:8-13.

42. Ferraccioli GF, Bambara LM, Ferraris M, Perpignano G, Cattaneo R, Porzio F, Accardo S, Mattara L, Zoppini A, Benucci M, Ostuni PA, Pasero G. Effects of cyclosporin on joint damage in rheumatoid arthritis. The Italian Rheumatologists Study Group on Rheumatoid Arthritis. Clin Exp Rheumatol. 1997;15 Suppl 17:S83-9.

43. Goldbach-Mansky R, Wilson M, Fleischmann R, Olsen N, Silverfield J, Kempf P, Kivitz A, Sherrer Y, Pucino F, Csako G, Costello R, Pham TH, Snyder C, van der Heijde D, Tao X, Wesley R, Lipsky PE. Comparison of Tripterygium wilfordii Hook $F$ versus sulfasalazine in the treatment of rheumatoid arthritis: a randomized trial. Ann Intern Med. 2009;151(4):229-40.

44. Hannonen P, Möttönen T, Hakola M, Oka M. Sulfasalazine in early rheumatoid arthiritis. Arthritis Rheum. 1993;36(11):1501-9.

45. Hetland ML, Stengaard-Pedersen K, Junker P, Lottenburger T, Ellingsen T, Andersen LS, Hansen I, Skjødt H, Pedersen JK, Lauridsen UB, Svendsen A, Tarp U, Pødenphant J, Hansen G, Lindegaard H, de Carvalho A, Østergaard M, Hørslev-Petersen K, CIMESTRA Study Group. Combination treatment with methotrexate, cyclosporine, and intraarticular betamethasone compared with methotrexate and intraarticular betamethasone in early active rheumatoid arthritis: an investigator-initiated, multicenter, randomized, double-blind, parallel-group, placebo-controlled study. Arthritis Rheum. 2006;54(5):1401-9.

46. Hetland ML, Stengaard-Pedersen K, Junker P, Lottenburger T, Hansen I, Andersen LS, Tarp U, Svendsen A, Pedersen JK, Skjødt H, Lauridsen UB, Ellingsen T, Hansen GV, Lindegaard H, Vestergaard A, Jurik AG, Østergaard M, Hørslev-Petersen K, CIMESTRA study group. Aggressive combination therapy with intra-articular glucocorticoid injections and conventional disease-modifying anti-rheumatic drugs in early rheumatoid arthritis: second-year clinical and radiographic results from the CIMESTRA study. Ann Rheum Dis. 2008;67(6):815-22.

47. Landewé $R B$, Boers $M$, Verhoeven $A C$, Westhovens $R$, van de Laar MA, Markusse HM, van Denderen JC, Westedt ML, Peeters AJ, Dijkmans BA, Jacobs P, Boonen A, van der Heijde DM, van der Linden S. COBRA combination therapy in patients with early rheumatoid arthritis: longterm structural benefits of a brief intervention. Arthritis Rheum. 2002; 46(2):347-56.

48. Larsen A, Kvien TK, Schattenkirchner M, Rau R, Scott DL, Smolen JS, Rozman B, Westhovens R, Tikly M, Oed C, Rosenburg R, European Leflunomide Study Group. Slowing of disease progression in rheumatoid arthritis patients during long-term treatment with leflunomide or sulfasalazine. Scand J Rheumatol. 2001;30(3):135-42.

49. Maravic M, Bologna C, Daures JP, Jorgensen C, Combe B, Sany J. Radiologic progression in early rheumatoid arthritis treated with methotrexate. J Rheumatol. 1999;26(2):262-7.
50. Marchesoni A, Battafarano N, Arreghini M, Pellerito R, Cagnoli M, Prudente P Cerase A, Priolo F, Tosi S. Step-down approach using either cyclosporin A or methotrexate as maintenance therapy in early rheumatoid arthritis. Arthritis Rheum. 2002:47(1):59-66.

51. Peltomaa R, Paimela L, Helve T, Leirisalo-Repo M. Comparison of intramuscular gold and sulphasalazine in the treatment of early rheumatoid arthritis A one year prospective study. Scand I Rheumatol. 1995;24(6):330-5

52. Sanmarti R, Gomez A, Ercilla G, Gratacos J, Larrosa M, Suris X, Vinas O, Salvador G, Munoz-Gomez J, Canete JD. Radiological progression in early rheumatoid arthritis after DMARDS: a one-year follow-up study in a clinical setting. Rheumatology (Oxford). 2003;42(9):1044-9.

53. Sarzi-Puttini $P, D^{\prime}$ Ingianna E, Fumagalli $M$, Scarpellini $M$, Fiorini T, ChériéLignière EL, Panni B, Fiorentini F, Corbelli V, Beyene NB, Mastaglio C, Severi C, Locati M, Cazzola M, Menozzi G, Monti G, Saccardo F, Alfieri $G$, Atzeni F. An open, randomized comparison study of cyclosporine A, cyclosporine $\mathrm{A}+$ methotrexate and cyclosporine $\mathrm{A}+$ hydroxychloroquine in the treatment of early severe rheumatoid arthritis. Rheumatol Int. 2005;25(1):15-22.

54. Scott DL, Dawes PT, Fowler PD, Grindulis KA, Shadforth M, Bacon P. Antirheumatic drugs and joint damage in rheumatoid arthritis. Q J Med. 1985; 54(213):49-59.

55. Scott DL, Dawes PT, Tunn E, Fowler PD, Shadforth MF, Fisher J, Clarke S, Collins M, Jones P, Popert AJ, et al. Combination therapy with gold and hydroxychloroquine in rheumatoid arthritis: a prospective, randomized, placebo-controlled study. Br J Rheumatol. 1989;28(2):128-33.

56. Scott DL, Greenwood A, Davies J, Maddison PJ, Maddison MC, Hall ND. Radiological progression in rheumatoid arthritis: do D-penicillamine and hydroxychloroquine have different effects? Br J Rheumatol. 1990;29(2):126-7.

57. Smith PR, Brown GM, Meyers OL. An open comparative study of auranofin vs. gold sodium thiomalate. J Rheumatol. 1982;8:190-6.

58. St Clair EW, van der Heijde DM, Smolen JS, Maini RN, Bathon JM, Emery P, Keystone E, Schiff M, Kalden JR, Wang B, Dewoody K, Weiss R, Baker D, Active-Controlled Study of Patients Receiving Infliximab for the Treatment of Rheumatoid Arthritis of Early Onset Study Group. Combination of infliximab and methotrexate therapy for early rheumatoid arthritis: a randomized, controlled trial. Arthritis Rheum. 2004:50(11):3432-43.

59. Strand V, Cohen S, Schiff M, Weaver A, Fleischmann R, Cannon G, Fox R, Moreland L, Olsen N, Furst D, Caldwell J, Kaine J, Sharp J, Hurley F, Loew-Friedrich I. Treatment of active rheumatoid arthritis with leflunomide compared with placebo and methotrexate. Leflunomide Rheumatoid Arthritis Investigators Group. Arch Intern Med. 1999; 159(21):2542-50.

60. Svensson B, Pettersson H. Reumacon (CPH82) showed similar x-ray progression and clinical effects as methotrexate in a two year comparative study on patients with early rheumatoid arthritis. Scand J Rheumatol. 2003; 32(2):83-8.

61. Svensson B, Boonen A, Albertsson K, van der Heijde D, Keller C, Hafström I. Low-dose prednisolone in addition to the initial disease-modifying antirheumatic drug in patients with early active rheumatoid arthritis reduces joint destruction and increases the remission rate: a two-year randomized trial. Arthritis Rheum. 2005;52(11):3360-70.

62. Van Riel PL, Larsen A, Van de Putte LB, Gribnau FW. Effects of aurothioglucose and auranofin on radiographic progression in rheumatoid arthritis. Clin Rheumatol. 1986;5(3):359-64.

63. Wassenberg $\mathrm{S}$, Rau R, Steinfeld P, Zeidler H. Very low-dose prednisolone in early rheumatoid arthritis retards radiographic progression over two years: a multicenter, double-blind, placebo-controlled trial. Arthritis Rheum. 2005; 52(11):3371-80

64. Bruynesteyn $\mathrm{K}$, van der Heijde D, Boers $\mathrm{M}$, et al. Determination of the minimal clinically important difference in rheumatoid arthritis joint damage of the Sharp/van der Heijde and Larsen/Scott scoring methods by clinical experts and comparison with the smallest detectable difference. Arthritis Rheum. 2002;46(4):913-20.

65. Sokka T. Radiographic scoring in rheumatoid arthritis: a short introduction to the methods. Bull NYU Hosp Jt Dis. 2008;66(2):166-8.

66. Morgenstern $\mathrm{H}$. Uses of ecologic analysis on epidemiologic research. Am J Public Health. 1982;72(12):1336-43.

67. Berlin JA, Santanna J, Schmid CH, Szczech LA, Feldman HI. Individual patient-versus group-level meta-regression for the investigation of 
treatment effect modifiers: ecological bias rears its ugly head. Stat Med. 2002;21(3):371-87.

68. Rahman MU, Buchanan J, Doyle MK, Hsia EC, Gathany T, Parasuraman S, Aletaha D, Matteson EL, Conaghan PG, Keystone E, van der Heijde D,

Smolen JS. Changes in patient characteristics in anti-tumour necrosis factor clinical trials for rheumatoid arthritis: results of an analysis of the literature over the past 16 years. Ann Rheum Dis. 2011;70:1631-40.

Submit your next manuscript to BioMed Central and we will help you at every step:

- We accept pre-submission inquiries

- Our selector tool helps you to find the most relevant journal

- We provide round the clock customer support

- Convenient online submission

- Thorough peer review

- Inclusion in PubMed and all major indexing services

- Maximum visibility for your research

Submit your manuscript at www.biomedcentral.com/submit
O BioMed Central 